\title{
Kundenbindung in Handels- und Serviceunternehmen - Die Wirkung von Kundenbindungsinstrumenten auf Einstellungen und Kaufverhalten
}

\author{
Thomas Rudolph • Liane Nagengast
}

Eingegangen: 15. April 2012 / Angenommen: 12. November 2012 /

Online publiziert: 11. Dezember 2012

(C) Wirtschaftsuniversität Wien, Austria 2012

Zusammenfassung Um sich vom Wettbewerb zu differenzieren, setzen Marketingmanager unterschiedliche Kundenbindungsinstrumente ein. Gerade im Rahmen von Loyalitätsprogrammen bieten sich für Handels- und Serviceunternehmen heute vielfältige Möglichkeiten zur individuellen Kundenansprache. Die damit einhergehenden Investitionen sind allerdings nicht immer von Erfolg gekrönt, wie der Literaturüberblick zeigt. Dies liegt vor allem daran, dass Marketingentscheider unterschiedliche Wirkungsweisen bestimmter Instrumente zu wenig berücksichtigen. Der Einsatz erfolgt oft willkürlich und die Instrumente sind zu wenig auf die jeweiligen Bedingungen und die entsprechenden Zielgruppen abgestimmt. Vor diesem Hintergrund gibt der Beitrag einen Überblick zum Thema „Kundenbindungsinstrumente“. Er zeigt eine umfassende Klassifizierung auf und begründet die Wirkungsweise unterschiedlicher Kundenbindungsinstrumente aus motivationstheoretischer Sicht. Auf Basis empirischer Erkenntnisse beschreibt er die Wirkung von Kundenbindungsinstrumenten unter verschiedenen Bedingungen und leitet schliesslich Implikationen für das Management zum effektiven und effizienten Einsatz von Kundenbindungsinstrumenten ab. Abschliessend werden offene Forschungsfelder in diesem Bereich diskutiert.

Schlüsselwörter Beziehungsmarketing · Kundenbindung ·

Kundenbindungsinstrumente · Loyalitätsprogramme · Intrinsische Motivation · Extrinsische Motivation

\section{JEL Klassifikationen M31}

T. Rudolph $(\bowtie) \cdot$ L. Nagengast

Forschungszentrum für Handelsmanagement, Universität St. Gallen, Dufourstrasse 40a, 9000 St. Gallen, Schweiz

e-mail: Thomas.Rudolph@unisg.ch

L. Nagengast

e-mail: Liane.Nagengast@unisg.ch 


\begin{abstract}
In order to differentiate from their competitors, marketing managers increasingly count on relationship marketing instruments. As customer loyalty programs have gained importance during the last decade, retailers face various new possibilities to reach their customers on a one-to-one level. However, empirical evidence in this field shows that the enormous investments do not always yield the desired effects. One reason is that marketing managers disregard that the effectiveness of different instruments might depend on particular conditions, target groups, and situations. The article gives an overview about the influence of relationship marketing instruments on customers' attitudes and behaviors. It provides a holistic classification of relationship marketing instruments and explains the effectiveness from a theoretical perspective. We summarize empirical studies dealing with the effectiveness of different instruments under certain conditions. Furthermore, we derive implications for retail managers about how to successfully use relationship marketing instruments. Finally, we discuss directions for future research.
\end{abstract}

Keywords Relationship marketing · Relationship marketing instruments · Loyalty programs · Direct marketing · Intrinsic motivation · Extrinsic motivation

\title{
1 Einleitung
}

\subsection{Hohe Investitionen in Kundenbindungsinstrumente}

Um ein möglichst hohes Mass an Kundenzufriedenheit und -loyalität zu erreichen, greifen Manager in Handels- und Serviceunternehmen auf unterschiedliche Marketing- und Profilierungsinstrumente zurück (Ailawadi und Keller 2004, S. 333340; Dabholkar et al. 1996, S. 11-15; Gomez et al. 2004, S. 270; Grewal et al. 2009, S. 2; Kunkel und Berry 1968, S. 26; Pan und Zinkhan 2006, S. 230-232). In den vergangenen ein bis zwei Jahrzehnten haben sich das generelle Marketingverständnis sowie die Art der eingesetzten Instrumente von einer transaktionsorientierten zu einer beziehungsorientierten Sichtweise gewandelt (z.B. Berry 1995, S. 236; Brodie et al. 1997, S. 383; Bruhn 2009, S. 9f; Grönroos 1999, S. 327-334; Hansen 2011, S. 295; Palmatier et al. 2006; Reinartz und Krafft 2001, S. 1264; Shugan 2005, S. 185f). Während früher eine kurzfristige Sichtweise vorherrschte, bei der Einzeltransaktionen und die Akquisition neuer Kunden im Vordergrund standen, geht es heute um die langfristig orientierte Kundenbindung und Pflege von Kundenbeziehungen (z.B. Hansen 2011, S. 295; Hennig-Thurau und Hansen 2000, S. 5; Shugan 2005, S. 185). Daher setzen Unternehmen zunehmend sog. ,Kundenbindungsinstrumente“ (engl.: „,relationship marketing instruments“) ein, um die Einstellungen und das Kaufverhalten ihrer Kunden positiv zu beeinflussen (Bhattacharya und Bolton 2000, S. 327-354; Hennig-Thurau und Hansen 2000, S. 8-11; Grönroos 1999, S. 333; Verhoef 2003, S. 30). Gerade in Zeiten, in denen das angebotene Leistungsspektrum der Anbieter oftmals austauschbar ist, kommt solchen Instrumenten eine besondere Bedeutung zu, da sie die einzige Möglichkeit zur Differenzierung vom Wettbewerb darstellen (Berry 1995, S. 236; Bolton et al. 2004, S. 271; de Wulf et al. 2001, S. 33; Hennig-Thurau und Hansen 2000, S. 5). De Wulf et al. stellen daher fest: „Today’s retailers increasingly offer comparable merchandise, copy competitors' price promotions, share 
common distribution systems, and treat customers well in terms of services offered, so there are increased opportunities for directing greater attention to developing and implementing relationship marketing tactics“ (de Wulf et al. 2001, S. 45f).

Durch die zunehmende Verbreitung von Loyalitätsprogrammen und die damit einhergehende Möglichkeit zur personalisierten Kundenansprache, hat der Einsatz von Kundenbindungsinstrumenten in den vergangenen Jahren zugenommen. Insbesondere die Handelsbranche legte dabei stark zu (Deutscher Dialogmarketingverband 2011). Im Bereich Fast Moving Consumer Goods (FMCG) stiegen bspw. in den DACH-Ländern die Investitionen in Direktmarketingaktivitäten kontinuierlich an (Schweizer Direktmarketing Verband 2011). Auch die folgenden Zahlen spiegeln die zunehmende Ausrichtung der Marketingaktivitäten auf die Kundenbindung wider: In einer Managerbefragung deutscher Marketingentscheider gaben knapp $80 \%$ an, dass sie planen, neue Kundenbindungsprogramme aufzubauen oder ihre bestehenden Programme auszubauen. $46 \%$ der befragten Unternehmen wollten die Investitionen in Kundenbindungsprogramme erhöhen. Nach Ansicht der befragten Manager war die Kundenbindung damit der wichtigste Erfolgsfaktor im Jahr 2010 (BBDO 2009; NRF und KPMG 2011). Ein Blick in die USA macht die immensen Investitionen in Kundenbindungsaktivitäten und Loyalitätsprogramme noch deutlicher: Im Jahr 2009 wurden hier 1.8 Mrd. individuelle Mitgliedschaften in Loyalitätsprogrammen registriert, während es 2006 noch 1.3 Mrd. und im Jahr 2000 erst 0.9 Mrd. waren. Diese Zahl entspricht ca. sechsmal der US-Bevölkerung. Das heisst, jeder US-Bürger, vom Kleinkind bis zum Greis, gehörte im Schnitt sechs Loyalitätsprogrammen an. Jeder Haushalt nahm demnach an durchschnittlich 14.1 unterschiedlichen Loyalitätsprogrammen teil (Ferguson und Hlavinka 2007, 2009). Etwa 153 Mio. dieser Mitgliedschaften entfielen allein auf den Lebensmittelhandel (Ferguson und Hlavinka 2009).

\subsection{Fragliche Erfolgswirkung von Kundenbindungsinstrumenten}

Trotz dieser enormen Investitionen ist die Effizienz von Loyalitätsprogrammen und Kundenbindungsinstrumenten fraglich, da die hohen Implementierungskosten nur selten durch Mehrumsätze gedeckt werden (Diller 1997, S. 33; O’Malley 1998, S. 52f; Rigby et al. 2002, S. 101f). Dowling und Uncles (1997) stehen Loyalitätsprogrammen kritisch gegenüber: ,They might help to protect incumbents and might be regarded as a legitimate part of the marketer's armory, but at the cost of increasing marketing expenditures“ (Dowling und Uncles 1997, S. 71). Die wissenschaftliche Literatur führt zahlreiche Gründe für den oftmals ausbleibenden Erfolg von Loyalitätsprogrammen und Kundenbindungsinstrumenten an: Sie sind kostenintensiv, können einfach von Wettbewerbern kopiert werden, erhöhen die Preissensitivität der Konsumenten und schaden damit letztlich der Loyalität (Henderson et al. 2011; Melancon et al. 2011, S. 341f; Nunes und Dréze 2006, S. 129f; O’Malley 1998, S. $52-$ 55; Yi und Jeon 2003, S. 230). Hinzu kommt, dass die Wirkung oft nur kurzfristig ist und mit Wegnahme bestimmter Anreize auch der gewünschte Effekt auf die Einstellungen und das Kaufverhalten der Konsumenten wieder verschwindet. Auch führen Loyalitätsprogramme und Kundenbindungsinstrumente oftmals nicht zu einer Loyalität gegenüber dem Anbieter, sondern fördern lediglich die Loyalität zum Loyalitätsprogramm und seinen Anreizen (Dowling und Uncles 1997; Evanschitzky et al. 2012; 
Meyer-Waarden 2007; Yi und Jeon 2003). Ohne das Loyalitätsprogramm mit seinen entsprechenden Anreizen verschwindet auch die Loyalität wieder. Die Folge aus den beschriebenen Mechanismen ist, dass Unternehmen Kundenbindungsinstrumente aktionistisch in kurzen Abständen einsetzen, um die Konsumenten permanent zu beeinflussen oder auf Marketingaktivitäten von Wettbewerbern zu reagieren (Deci et al. 1999, S. 627; Yi und Jeon 2003, S. 229). Die Instrumente sind dabei selten explizit durchdacht und auf bestimmte Zielgruppen abgestimmt (Kumar und Shah 2004, S. 318-321).

\subsection{Zielsetzung und Vorgehen des Artikels}

Vor diesem Hintergrund gibt der Beitrag einen Überblick zum aktuellen Stand der Forschung im Bereich der Kundenbindungsinstrumente. Dabei stehen zwei Hauptziele im Vordergrund:

1. Auf Basis bestehender Forschungsergebnisse werden Implikationen zum erfolgreichen Einsatz unterschiedlicher Kundenbindungsinstrumente abgeleitet. Marketingmanager erhalten so vielfältige Gestaltungshinweise für ihre Kundenbindungsbzw. Loyalitätsprogramme.

2. Der Artikel gibt Forschern auf diesem Gebiet einen Überblick und dient als Einstiegslektüre. Zudem zeigt er zukünftig bedeutende Forschungspfade auf.

Um diese Ziele zu erreichen, finden sich in Abschn. 2 zunächst eine Begriffsabgrenzung sowie eine Systematisierung der Kundenbindungsinstrumente. AnschlieBend zeigt Abschn. 3 unterschiedliche Auswirkungen auf die Einstellungen und das Kaufverhalten aus konsumententheoretischer Sicht auf. Auch potenzielle negative Effekte und Moderatoren der angesprochenen Zusammenhänge werden diskutiert. Abschnitt 4 beschreibt die möglichen Ausprägungen der Kundenbindungsinstrumente im Detail und stellt empirische Forschungsergebnisse zur Wirkungsweise dar. Abschnitt 5 gibt konkrete Hinweise für das Management zum effektiven und effizienten Einsatz von Kundenbindungsinstrumenten. Abschnitt 6 fasst den Beitrag zusammen, gibt Implikationen für künftige Forschungsprojekte und weist auf Limitationen hin (vgl. Abb. 1).

\section{Begriffsabgrenzung und Systematisierung von Kundenbindungsinstrumenten}

\subsection{Definitorische Abgrenzung von Kundenbindungsinstrumenten}

Der Begriff „Beziehungsmarketing“ bezeichnet generell alle Aktivitäten eines Unternehmens, die darauf ausgerichtet sind, Kundenbeziehungen aufzubauen, zu entwickeln oder zu pflegen (Bolton et al. 2004; Homburg und Bruhn 2010, S. 8; Morgan und Hunt 1994, S. 22; vgl. auch Leusser et al. 2011, S. 19f für eine Abgrenzung zu verwandten Konstrukten). Das zentrale Ziel des Beziehungsmarketing besteht in der Kundenbindung (Diller 1996, S. 81). Die hierbei zum Einsatz kommenden Kundenbindungsinstrumente sind entsprechend definiert als Marketingaktivitäten zum Aufbau und zur Pflege von Kundenbeziehungen (Bhattacharya und Bolton 2000, S. 328). 


\section{Einleitung}

\begin{tabular}{|l|l|l|l|l|l|l|l}
\hline $\begin{array}{l}1.1 \text { Hohe Investitionen in } \\
\text { Kundenbindungsinstrumente }\end{array}$ & $\begin{array}{l}1.2 \text { Fragliche Erfolgswirkung von } \\
\text { Kundenbindungsinstrumenten }\end{array}$ \\
\hline
\end{tabular}

2. Begriffsabgrenzung und Systematisierung von Kundenbindungsinstrumenten

\begin{tabular}{|l|l|l|}
\hline $\begin{array}{l}2.1 \text { Definitorische Abgrenzung von } \\
\text { Kundenbindungsinstrumenten }\end{array}$ & $\begin{array}{l}2.2 \text { Begrifliche Abgrenzung zu } \\
\text { verwandten Forschungsgebieten }\end{array}$ & $\begin{array}{l}2.3 \text { Klassifizierung von } \\
\text { Kundenbindungsinstrumenten }\end{array}$ \\
\hline
\end{tabular}

3. Die Wirkung von Kundenbindungsinstrumenten aus theoretischer Sicht

\begin{tabular}{|l|l|l|l|}
\hline $\begin{array}{l}3.1 \text { Extrinsische und } \\
\text { intrinsische Motivation }\end{array}$ & $\begin{array}{l}3.2 \text { Wahrgenommene Selbst- } \\
\text { bestimmung von Konsumenten }\end{array}$ & $\begin{array}{l}3.3 \text { Langfristige } \\
\text { Auswirkungen von Anreizen }\end{array}$ & $\begin{array}{l}3.4 \text { Moderatoren der Wirkung von } \\
\text { Kundenbindungsinstrumenten }\end{array}$ \\
\hline
\end{tabular}

4. Empirische Forschungsergebnisse zur Wirkung von Kundenbindungsinstrumenten

\begin{tabular}{|l||l|l|l|}
\hline \hline $\begin{array}{l}4.1 \text { Monetäre vs. nicht- } \\
\text { monetare Anreize }\end{array}$ & $\begin{array}{l}\text { 4.2 Materielle vs. nicht- } \\
\text { materiele Anreize }\end{array}$ & $\begin{array}{l}\text { 4.3 Anbieterbezogene vs. nicht- } \\
\text { anbeterbezogene Anreize }\end{array}$ & $\begin{array}{l}4.4 \text { Direkte vs. indirekte } \\
\text { Anreize }\end{array}$ \\
\hline \begin{tabular}{l|ll}
4.5 Sofortige vs. verzögerte \\
Anreize
\end{tabular} & $\begin{array}{l}4.6 \text { Informative vs. } \\
\text { einschrankende Anreize }\end{array}$ & $\begin{array}{l}4.7 \text { Beziehungs- vs. } \\
\text { Verhaltensorientierte Anreize }\end{array}$ & $\begin{array}{l}4.8 \text { Unterschiedliche } \\
\text { Kommunikationsarten }\end{array}$ \\
\hline
\end{tabular}

5. Diskussion zum Einsatz von Kundenbindungsinstrumenten aus praktischer Sicht

\begin{tabular}{|l|l|l|l|l|}
\hline \hline $\begin{array}{l}5.1 \text { Ausgestaltung } \\
\text { der Instrumente }\end{array}$ & $\begin{array}{l}5.2 \text { Verhindern von } \\
\text { Cherry-Picking-Verhalten }\end{array}$ & $\begin{array}{l}5.3 \text { Fördern von Verbunden- } \\
\text { statt Gebundenheit }\end{array}$ & $\begin{array}{l}5.4 \text { Abstimmung auf } \\
\text { Situationen \& Zielgruppen }\end{array}$ & $\begin{array}{l}5.5 \text { Effizienter } \\
\text { Einsatz }\end{array}$ \\
\hline
\end{tabular}

\section{Ausblick und Fazit}

6.1 Künftige Forschungsarbeiten zu Kundenbindungsinstrumenten

6.2 Zusammenfassung und Fazit

Abb. 1 Überblick des Artikels

Unternehmen greifen häufig auf bestimmte Anreize und Belohnungen im Rahmen von Loyalitätsprogrammen zurück. Ein wesentlicher Unterschied von Kundenbindungsinstrumenten zu Marketinginstrumenten im Allgemeinen besteht in der individuellen Kundenansprache im Gegensatz zu einem ungerichteten „Massenmarketing“ (Berry 1995, S. 236; Grönroos 1996, S. 11f; Hennig-Thurau und Hansen 2000, S. 5f). Die Palette der eingesetzten Anreize ist breit und reicht von monetären Anreizen, wie Coupons und Preisaktionen, über materielle Geschenke, wie Kundenzeitschriften und Werbegeschenke, bis zu immateriellen Anreizen, wie Einladungen zu Veranstaltungen und Events (Bolton et al. 2004, S. 279; Helm und Ludl 2005, S. 1137-1139; Kumar und George 2007, S. 163f; Lemon et al. 2001, S. 22f; Reinartz 2010, S. 411416; Rust et al. 2004, S. 112).

Mit dem Einsatz dieser Kundenbindungsinstrumente verfolgen Handelsmanager bestimmte Ziele, wie z.B. eine Verbesserung ihres Images, eine Steigerung der Kundenzufriedenheit, das Anregen von Cross-Selling, eine Erhöhung der Einkaufshäufigkeit und damit einhergehend eine Erhöhung des Kundenwertes bzw. „Customer Lifetime Values“ (Gàzquez-Abad et al. 2011, S. 168f). Im Kern geht es darum, die Kundenbindung nachhaltig zu stärken und damit einen Mehrwert für den Konsumenten und den Anbieter zu schaffen. Rust et al. sprechen in diesem Zusammenhang von „Retention Equity“ bzw. „Relationship Equity“ (Rust et al. 2000, S. 95-113). 
Sie zeigen, dass es heute häufig nicht mehr gelingt, Kunden allein durch eine starke (Handels-)Marke (,Brand Equity“) oder ein besonders gutes Angebot (,,Value Equity") an ein Unternehmen zu binden. Vielmehr sollten Anbieter den Konsumenten bestimmte Benefits bzw. Belohnungen anbieten und sie so motivieren, auch ihrerseits Investitionen in die Beziehung zu tätigen. Kundenbindungsinstrumente setzen genau hier an. Sie belohnen den Kunden bspw. in monetärer oder emotionaler Form und motivieren ihn so, eine Gegenleistung zu erbringen und Investitionen in die Beziehung zu tätigen. Diese positive Beeinflussung der „Relationship Equity“ bildet schliesslich die Grundlage der Loyalität der Konsumenten (Vogel et al. 2008).

\subsection{Begriffliche Abgrenzung zu verwandten Forschungsgebieten}

In den vergangenen ein bis zwei Jahrzehnten kamen zahlreiche Begriffe auf, welche die Thematik der individuellen Kundenansprache behandeln und vom Beziehungsmarketing und der Kundenbindung definitorisch abzugrenzen sind.

So bezeichnet das Direktmarketing personalisierte Kommunikationsmaßnahmen (z.B. Newsletter, Mailing, Anruf), die z.T. auch eine gewisse Interaktivität der Kunden erfordern (Mann 2007, S. 2f). Im Fokus des Direktmarketings stehen kommunikations- und distributionspolitische Aspekte des Beziehungsmarketings (Hesse et al. 2007, S. 6). Im Unterschied dazu lässt sich das Beziehungsmarketing und die damit verbundenen Kundenbindungsaktivitäten allen vier Bereichen des Marketing-Mix zuordnen und beschränkt sich nicht auf die Kommunikation und Distrubition (Hansen 2011, S. 297). Ihm liegt somit eine umfassendere Sichtweise zugrunde.

Auch der Begriff der „Individualisierung“ tritt häufig im Zusammenhang mit der Kundenbindung auf oder wird im ähnlichen Kontext verwendet und ist daher abzugrenzen. Im Unterschied zur Kundenbindung als umsetzungs- und zielorientiertes Konzept, beschreibt die Individualisierung ein generelles Prinzip, welches im Beziehungsmarketing - neben weiteren Prinzipien (Diller 1995, S. 443f) - von Bedeutung ist. Im Rahmen der Kundenbindungsaktivitäten äussert sich das Prinzip der Individualisierung bspw. durch ein auf die Zielkunden zugeschnittenes Leistungsangebot oder massgeschneiderte Kommunikationsaktivitäten (Diller 1995, S. 443; Georgi und Mink 2011, S. 75).

Auch der Begriff des „Customer Relationship Management (CRM)“ wird oft im Zusammenhang oder sogar als Synonym für „Kundenbindung“ genannt (Parvatiyar und Sheth 2001, S. 4). Aufgrund der Herkunft des Begriffs aus der IT-Branche, liegt ihm eine prozessorientierten Sichtweise zugrunde (Payne und Frow 2005, S. 167; Ryals und Payne 2001, S. 3). Kumar und Reinartz (2006) definieren CRM daher wie folgt: „CRM is the practice of analyzing and utilizing marketing databases and leveraging communication technologies to determine corporate practices and methods that will maximize the lifetime value of each individual customer to the firm" (Kumar und Reinartz 2006, S. 5). Aus dieser Definition geht hervor, dass beim CRM eher der Prozess der Daten- und Informationsgewinnung sowie die damit einhergehende Ableitung von Massnahmen zur Kundenbindung im Vordergrund stehen. Hierbei liegt der Fokus zum einen auf den technologischen Aspekten der Informationsgewinnung und zum anderen auf der optimalen Verteilung des Marketingbudgets auf besonders wertvolle Kundensegmente (Heiligenthal und Skiera 2007, S. 118). 
Der vorliegende Artikel fokussiert sich auf die Kundenbindung als Gesamtheit der Aktivitäten eines Unternehmens, die darauf ausgerichtet sind, Kundenbeziehungen aufzubauen, zu entwickeln und zu pflegen. Hierbei stehen die Instrumente im Vordergrund, die der Beibehaltung und Intensivierung von Kundenbeziehungen dienen (Bruhn 2011, S. 414). Mit anderen Worten sind die betrachteten Instrumente auf bestehende Kundenbeziehungen ausgerichtet. Es geht dabei nicht um die Akquisition von Neukunden oder die Rückgewinnung abgewanderter Kunden (Bruhn 2009, S. 60; Leusser et al. 2011, S. 19f).

\subsection{Klassifizierungen von Kundenbindungsinstrumenten}

Kundenbindungsinstrumente lassen sich in Hinblick auf unterschiedliche Kriterien einteilen. In der wissenschaftlichen Literatur finden sich unterschiedliche Klassifizierungsschemata, die sich z.T. ähneln und nicht immer überschneidungsfrei sind (z.B. Bhattacharya und Bolton 2000; Bolton et al. 2004; Diller 1995, S. 445; Diller et al. 2005; Dowling und Uncles 1997; Homburg und Bruhn 2010; Puchner 2011; Reinartz und Kumar 2000, S. 172-175). Diese Systematisierungen beleuchten meist punktuell einige wenige Kriterien. Sie betrachten und unterteilen einzelne ausgewählte Instrumente anhand bestimmter Merkmale (z.B. materielle vs. nichtmaterielle Anreize), ohne jedoch eine differenzierte Gesamtschau vorzunehmen. Eine ganzheitliche Klassifizierung, die den aktuellen Stand der Forschung abbildet und auf Basis bestehender Erkenntnisse die Instrumente systematisch hinsichtlich ihrer Charakteristika einteilt, existiert bisher nicht. Der vorliegende Artikel sammelt und kombiniert daher sowohl bestehende konzeptionelle Klassifizierungen (z.B. Bhattacharya und Bolton 2000; Bolton et al. 2004; Dowling und Uncles 1997; Homburg und Fassnacht 2001) als auch empirische Studien, wie Experimente, Kundenbefragungen und Längsschnittanalysen (z.B. Dholakia 2006; Melancon et al. 2011; Roehm et al. 2002). Er liefert ein holistischeres Bild als bestehende Typologisierungen aus Lehrbüchern und empirischen Studien. Somit ist es möglich, den aktuellen Stand der Forschung und das heutige Wissen über die Wirkung von Kundenbindungsinstrumenten aufzuzeigen und die bestehenden empirischen Ergebnisse dieser konzeptionellen Systematik zuzuordnen. Mithilfe dieser Gesamtschau lassen sich die beiden definierten Ziele erreichen und Empfehlungen für die Marketingpraxis sowie Hinweise für Forscher zu interessanten Phänomenen auf diesem Gebiet ableiten.

Zunächst gibt Tab. 1 einen Überblick der identifizierten Klassifizierungen und ordnet bestehende empirische Studien entsprechend zu. Die konkrete Beschreibung dieser Klassifizierungskriterien und ihrer Ausprägungen findet sich in Abschn. 4.

Obwohl die Kundenbindung generell langfristige Ziele verfolgt (z.B. Hansen 2011, S. 295; Hennig-Thurau und Hansen 2000, S. 5), zeichnen sich nicht alle der genannten Instrumente durch eine langfristige Wirkungsweise aus (Bruhn 2009, S. 135f; Shugan 2005, S. 186). Während bestimmte Instrumente eher kurzfristigen Aktionscharakter besitzen, wirken andere Instrumente langfristiger und nachhaltiger. Berry (1995) teilt die Instrumente daher entsprechend ihres Potenzials zur Generierung von langfristigen bzw. nachhaltigen Wettbewerbsvorteilen in Level-1-, Level-2-, und Level-3-Instrumente ein. Während Level-1-Instrumente eher kurzfristige Aktionsinstrumente (z.B. monetäre Anreize) meinen, zielen Level-2- und Level3-Instrumente auf eine langfristige Verbesserung der Beziehung zum Konsumenten 
Tab. 1 Klassifizierung von Kundenbindungsinstrumenten

\begin{tabular}{|c|c|c|}
\hline Klassifizierungskriterium & Ausprägungen & Beispielstudien \\
\hline Art des Nutzens & $\begin{array}{l}\text { Monetär vs. nicht-monetär bzw. } \\
\text { ökonomisch vs. sozial }\end{array}$ & $\begin{array}{l}\text { Helm und Ludl (2005); } \\
\text { Rust und Verhoef (2005); Müller } \\
\text { (2006); Puchner (2011); Melancon } \\
\text { et al. (2011) }\end{array}$ \\
\hline Tangibilität & Materiell vs. nicht-materiell & $\begin{array}{l}\text { de Wulf et al. (2001); } \\
\text { Roehm et al. (2002) }\end{array}$ \\
\hline Zeitliche Wirkung & Sofort vs. verzögert & $\begin{array}{l}\text { Zhang et al. (2000); } \\
\text { Tietje (2002); Yi und Jeon (2003); } \\
\text { Keh und Lee (2006) }\end{array}$ \\
\hline Nutzenstiftung & Direkt vs. indirekt & Yi und Jeon (2003) \\
\hline \multirow[t]{2}{*}{ Anbieterbezug } & $\begin{array}{l}\text { Anbieter- vs. } \\
\text { nicht-anbieterbezogen }\end{array}$ & $\begin{array}{l}\text { Roehm et al. (2002); } \\
\text { Tietje (2002); Keh und Lee (2006); } \\
\text { Puchner (2011) }\end{array}$ \\
\hline & $\begin{array}{l}\text { Firmenspezifisch vs. } \\
\text { firmenübergreifend }\end{array}$ & $\begin{array}{l}\text { Lemon und von Wangenheim } \\
\text { (2009); Müller (2006) }\end{array}$ \\
\hline Kontrollgrad & Informativ vs. einschränkend & $\begin{array}{l}\text { Dholakia (2006); Melancon et al. } \\
\text { (2011) }\end{array}$ \\
\hline Wirkungsweise & $\begin{array}{l}\text { Beziehungs- vs. } \\
\text { verhaltensorientiert }\end{array}$ & Gàzquez-Abad et al. (2011) \\
\hline Kommunikationsart & $\begin{array}{l}\text { Post, E-Mail, Telefon, mobile } \\
\text { Kommunikation }\end{array}$ & $\begin{array}{l}\text { Reutterer et al. (2006); } \\
\text { Godfrey et al. (2011) }\end{array}$ \\
\hline
\end{tabular}

ab (Berry 1995, S. 240f). Basierend auf der bestehenden Literatur (z.B. Berry 1995; Kumar und Shah 2004; Bhattacharya und Bolton 2000) kategorisieren wir daher die in empirischen Studien untersuchten Kundenbindungsinstrumente ebenfalls in kurzfristig und langfristig orientierte Instrumente. Da Kundenbindungsinstrumente stets auch aus einer Kombination bestimmter Ausprägungen bestehen können, ist die folgende Einteilung nicht allgemeingültig. Sie zeigt ein idealtypisches Bild der Wirkungsweise unterschiedlicher Instrumente auf und verdeutlicht, dass sich diese in ihrer Nachhaltigkeit unterscheiden (Tab. 2).

Da Kundenbindungsinstrumente unterschiedliche Wirkungen auf Kundenseite hervorrufen, gilt es je nach Zielsetzung unterschiedliche Instrumente einzusetzen. Um die unterschiedlichen Effekte besser zu verstehen, zeigt der folgende Abschnitt den theoretischen Hintergrund auf, der die Wirkungsweise von Kundenbindungsinstrumenten begründet.

\section{Die Wirkung von Kundenbindungsinstrumenten aus theoretischer Sicht}

\subsection{Extrinsische vs. intrinsische Motivation}

Aus theoretischer Sicht ist die Motivation, ein bestimmtes Verhalten (z.B. Kauf) auszuüben, entweder intrinsischer oder extrinsischer Natur (Deci 1971, S. 105; White 
Tab. 2 Kundenbindungsinstrumente mit kurz- und langfristiger Wirkungsweise

\begin{tabular}{lll}
\hline $\begin{array}{l}\text { Eher kurzfristig orientierte } \\
\text { Kundenbindungsinstrumente }\end{array}$ & $\begin{array}{l}\text { Können sowohl kurz- als auch } \\
\text { langfristig orientiert sein }\end{array}$ & $\begin{array}{l}\text { Eher langfristig orientierte } \\
\text { Kundenbindungsinstrumente }\end{array}$ \\
\hline $\begin{array}{l}\text { Monetäre Anreize } \\
\text { Materielle Anreize }\end{array}$ & Nicht-monetäre Anreize \\
Sofortige Anreize & Nicht-materielle Anreize \\
Direkte Anreize & Anbieterbezogene Anreize & Indirekte Anreize \\
& $\begin{array}{l}\text { Nicht-anbieterbezogene } \\
\text { Anreize }\end{array}$ & Firmenübergreifende Anreize \\
& Firmenspezifische Anreize & Beziehungsorientierte Anreize \\
Einschränkende Anreize & Informative Anreize & \\
Verhaltensorientierte Anreize & Anreize per Post, E-Mail, & Telefon, mobiler \\
& Kommunikation & \\
\hline
\end{tabular}

1959, S. 328-330). Intrinsische Motivation entsteht durch die Zufriedenheit, welche aus einem Verhalten selbst resultiert, wohingegen extrinsische Motivation durch bestimmte Anreize bzw. Belohnungen für ein Verhalten entsteht (Deci et al. 1999, S. 627; Ryan und Deci 2002, S. 10; Vallerand und Ratelle 2002, S. 42). Rein extrinsisch motivierte Individuen üben ein bestimmtes Verhalten nur aus, weil sie eine Belohnung dafür erhalten. Übertragen auf eine Anbieter-Kunden-Beziehung bedeutet dies, dass intrinsisch motivierte Kunden bei einem Anbieter kaufen, da hieraus Zufriedenheit oder andere positive Gefühle resultieren. Aus der positiven Einstellung gegenüber dem Händler entsteht die Motivation, immer wieder dort einzukaufen. Extrinsisch motivierte Kunden kaufen dagegen nur dann bei einem bestimmten Anbieter, wenn sie eine Belohnung dafür erhalten. Die Wirkung unterschiedlicher Kundenbindungsinstrumente lässt sich daher motivationstheoretisch begründen. Obwohl Kundenbindungsinstrumente aufgrund ihres Belohnungscharakters häufig in erster Linie auf die extrinsische Motivation einwirken, können sie auch die intrinsische Motivation (z.B. Zufriedenheit mit einem Anbieter) stimulieren: ,[...] certain tailored promotions - e.g., regional events - create positive affect that influences cumulative satisfaction with the organization“ (Bhattacharya und Bolton, S. 343).

In der Praxis sind - entgegen dieser idealtypischen Beschreibung - die eingesetzten Kundenbindungsinstrumente nicht immer eindeutig extrinsischen oder intrinsischen Anreizen zuzuordnen. Es existieren auch Mischformen, welche sowohl die extrinsische als auch die intrinsische Motivation beeinflussen. In diesem Fall gleichen sich die Wirkungen auf die Selbstbestimmung der Konsumenten - und folglich auf die extrinsische und intrinsische Motivation - zu einem gewissen Mass aus (Deci et al. 1999, S. 628). Denkbar sind bspw. materielle beziehungsorientierte Anreize (z.B. eine Schachtel Pralinen, ein Blumenstrauss als Dankeschön). Diese wirken durch den materiellen Charakter auf die extrinsische Motivation und durch den symbolischen Charakter auf die intrinsische Motivation (Zufriedenheit mit dem Anbieter) ein. 


\subsection{Wahrgenommene Selbstbestimmung von Konsumenten}

Eine Begründung für die unterschiedliche Wirkungsweise von Kundenbindungsinstrumenten liefert die „Cognitive Evaluation Theory“ (Deci 1975; Deci und Ryan 1985). Danach schränken verschiedene Anreizarten die wahrgenommene Selbstbestimmung der Konsumenten unterschiedlich stark ein. Bestimmte Anreize untergraben die Selbstbestimmung, indem sie die Konsumenten konkret zu einem bestimmten Verhalten auffordern und auf die extrinsische Motivation einwirken. Hierbei handelt es sich v.a. um monetäre Anreize, welche Händler einsetzen, um das Kaufverhalten zu beeinflussen (Bhattacharya und Bolton 2000; Bolton et al. 2004). Dabei unterliegt die Entscheidung des Kunden, sich loyal zu verhalten, rein ökonomischen Gesichtspunkten. Eine Beeinflussung der beziehungsbezogenen Einstellungen (z.B. Zufriedenheit, Vertrauen, Commitment) findet nicht statt. Einstellungsorientierte Anreize sind hingegen entweder rein informeller Natur oder erhöhen sogar die wahrgenommene Selbstbestimmung der Konsumenten. Sie wirken auf die intrinsische Motivation ein (Deci und Ryan 1985, S. 32). Das Ausmass, in dem Belohnungen die extrinsische bzw. intrinsische Motivation beeinflussen, ergibt sich folglich daraus, inwieweit sie die Selbstbestimmung fördern oder einschränken (Deci et al. 1999, S. 628; Dholakia 2006, S. 114; Graham 1994; Ryan und Deci 2002, S. 11f). Eine starke Einschränkung nehmen Anreize vor, die nur bei Erfüllung einer bestimmten Aufgabe wirksam werden (,task-contingent rewards"). Dagegen entfalten aufgabenunabhängige Anreize (,task-incontingent rewards") auch ohne Erfüllung einer Aufgabe ihre belohnende Wirkung und schränken die Selbstbestimmung weniger stark ein. Daneben existiert eine dritte Form, die sog. leistungsabhängigen Anreize (,,performance-contingent rewards"). Hier erhält ein Individuum nur eine Belohnung, wenn es eine Aufgabe besonders gut erfüllt oder ein bestimmtes Kriterium erreicht. Diese Anreize schränken die Selbstbestimmung am stärksten ein (Ryan et al. 1983, S. 738-741).

In Anbieter-Kunden-Beziehungen ist ein aufgabenabhängiger Anreiz z.B. ein Einkaufsgutschein. Erfüllt der Konsumenten die ,Aufgabe“ (Kauf) nicht, so ist der Gutschein wertlos. Ein Beispiel für einen leistungsorientierten Anreiz ist ein Gutschein, der zusätzlich an bestimmte Bedingungen (z.B. Gültigkeitsdatum, Mindesteinkaufsbetrag) geknüpft ist. Auch diese Belohnung ist direkt darauf ausgerichtet, ein bestimmtes Verhalten auszulösen und tritt nur in Kraft, wenn der Kunde die Aufgabe (Kauf) erfüllt und zudem eine bestimmte Leistung (z.B. Einkaufsbetrag) erbringt. Im Fall eines aufgabenunabhängigen Anreizes, wie einem Geschenk oder Dankesschreiben, entsteht ein Nutzen für den Kunden, ohne dass er eine Aufgabe erfüllen oder eine Leistung erbringen muss. Die wahrgenommene Selbstbestimmung bleibt unberührt und die intrinsische Motivation wird positiv beeinflusst (Bhattacharya und Bolton 2000, S. 343; Bolton et al. 2004, S. 280). Die Anpassung des Kaufverhaltens erfolgt indirekt über eine Einstellungsänderung.

\subsection{Langfristige Auswirkungen von Anreizen}

Forschungsarbeiten auf dem Gebiet der extrinsischen und intrinsischen Motivation haben sich intensiv mit den langfristigen Auswirkungen von Anreizen beschäftigt. 
Hierbei stand die Thematik im Vordergrund, wie extrinsische Anreize die intrinsische Motivation beeinflussen bzw. ob sich die intrinsische Motivation nach Wegnahme eines extrinsischen Anreizes verändert. Extrinsische (z.B. monetäre) Anreize können die intrinsische Motivation senken und die Einstellungen negativ beeinflussen (z.B. Deci 1971; Dholakia 2006, S. 110; vgl. hierzu die Meta-Analyse von Deci et al. 1999): „money [...] may act as a stimulus which leads the subjects to a cognitive reevaluation of the activity from one which is intrinsically motivated to one which is motivated primarily by the expectation of financial rewards. In short, money may work to „buy off“ one's intrinsic motivation for an activity“ (Deci 1971, S. 114). Nach Wegnahme eines Anreizes empfinden Individuen eine gewisse Enttäuschung und verspüren gleichzeitig ein Anrecht auf den Anreiz. Hinzu kommt, dass Personen oder Organisationen, die Geld für eine bestimmte Aufgabe bezahlen, diese Aufgabe offenbar als wenig befriedigend oder interessant ansehen. Diese Wahrnehmung überträgt sich auf das empfangende Individuum, welches die Aufgabe schliesslich auch nicht mehr befriedigend findet (Fehr und Falk 2002, S. 717). Besonders problematisch ist es, dass der Ersatz der intrinsischen durch die extrinsische Motivation langfristig anhält (Deci 1971, S. 114). Gemäss der Cognitive Evaluation Theory entsteht die langfristig negative Wirkung auch durch die wahrgenommene Einschränkung der Selbstbestimmung durch bestimmte Anreize. Individuen sehen ihr Bedürfnis nach Autonomie nicht mehr ausreichend befriedigt und die intrinsische Motivation wird langfristig untergraben (Fehr und Falk 2002, S. 714f). Im Unterschied zu extrinsischen Anreizen sind intrinsische Anreize nicht mit diesen Negativeffekten behaftet (Deci 1971, S. 114).

Erhalten intrinsisch motivierte Kunden, die aus Zufriedenheit immer wieder bei einem bestimmten Anbieter einkaufen, einen extrinsischen Anreiz (z.B. Einkaufsgutschein), so sinkt langfristig die intrinsische Motivation. Nach Wegnahme des Anreizes sind die Kunden folglich weniger mit dem Anbieter zufrieden als in der Ausgangssituation (Melancon et al. 2011, S. 357). Anbieter laufen Gefahr, die intrinsische Motivation durch einen extensiven Einsatz von extrinsisch orientierten Kundenbindungsinstrumenten zu untergraben (,,crowding out“") (Fehr und Falk 2002, S. 715).

\subsection{Moderatoren der Wirkung von Kundenbindungsinstrumenten}

Theoretische Wirkungsmechanismen liegen in der Realität häufig nicht in dieser idealtypischen Form vor. In Hinblick auf Kundenbindungsinstrumente zeigen Forschungsarbeiten, dass die Wirkung auf Einstellungen und Kaufverhalten je nach Situation und anvisierter Zielgruppe variiert. Bestimmte Bedingungen können die Wirkung beeinflussen, d.h. verstärken oder abschwächen (Bruhn 2009, S. 67 und S. 90). Nicht immer führt der Einsatz zu den postulierten positiven bzw. negativen Effekten. Während bestimmte Instrumente unter gewissen Bedingungen oder für bestimmte Kundensegmente positive Auswirkungen erzielen, ergeben sich unter anderen Bedingungen oder für andere Segmente keine oder sogar negative Effekte. Daher hat die Erforschung von zusätzlichen Einflussfaktoren (Moderatoren) auf die Wirkung von Kundenbindungsinstrumenten auf Einstellungen und Kaufverhalten an Bedeutung gewonnen. 


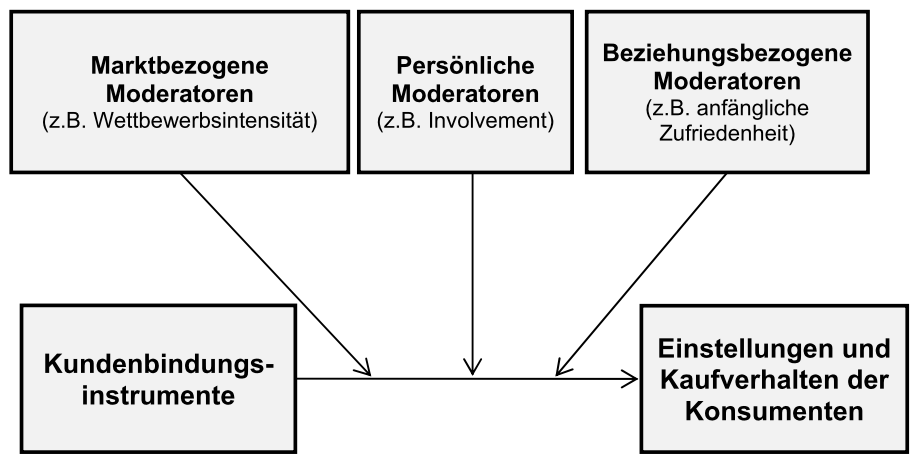

Abb. 2 Moderatoren der Wirkung von Kundenbindungsinstrumenten

Grundsätzlich sind zahlreiche Faktoren von Bedeutung, welche das Kaufverhalten der Konsumenten beeinflussen und daher zu Wechselwirkungen mit den Kundenbindungsinstrumenten führen können (Bolton et al. 2004, S. 274; Seiders et al. 2005, S. 27f). So beeinflussen spezifische Marktgegebenheiten (z.B. Wettbewerbssituation, Art der angebotenen Produkte) das Kaufverhalten der Konsumenten und können folglich mit der Wirkung der Kundenbindungsinstrumente interagieren (de Wulf et al. 2001, S. 44f; Jones et al. 2000, S. 65-267; Mittal und Kamakura 2001, S. 137; Seiders et al. 2005, S. 37f).

Auch Unterschiede in Hinblick auf die Beziehung des Konsumenten zum jeweiligen Anbieter (z.B. Länge der Beziehung, Zufriedenheit) beeinflussen das Kaufverhalten und können daher mit Kundenbindungsinstrumenten interagieren (z.B. Bolton 1998, S. 61; Bowman und Narayandas 2001, S. 292-294; Jones et al. 2000, S. 265267; Verhoef 2003, S. 38-41; Gàzquez-Abad et al. 2011, S. 170; Seiders et al. 2005, S. 37).

Zudem treten Unterschiede im Kaufverhalten auf, da sich die Konsumenten selbst hinsichtlich persönlicher Charakteristika oder Einstellungen (z.B. Einkommen, Involvement, Alter) unterscheiden (z.B. Homburg und Giering 2001, S. 54-57; Mägi 2003, S. 102-104; Mittal und Kamakura 2001, S. 137; Seiders et al. 2005, S. 37; Uncles et al. 2003, S. 298). Persönliche Charakteristika stellen daher einen weiteren potenziellen Moderator der Wirkung von Kundenbindungsinstrumenten dar.

Die beschriebenen Faktoren beeinflussen das Kaufverhalten direkt und können darüber hinaus mit weiteren Einflussfaktoren auf das Kaufverhalten interagieren (Seiders et al. 2005). Sie sind auch bei der Wirkung von Kundenbindungsinstrumenten auf die Einstellungen und das Kaufverhalten von Konsumenten zu berücksichtigen. Entsprechend unterteilen wir die Moderatoren der Wirkung von Kundenbindungsinstrumenten in die drei Kategorien: Marktbezogene, beziehungsbezogene und persönliche Moderatoren (Seiders et al. 2005, S. 27) (Abb. 2).

In Bezug auf marktbezogene Moderatoren fanden wissenschaftliche Forschungsarbeiten heraus, dass sich je nach betrachteter Branche die Wirkung von Kundenbindungsinstrumenten unterscheidet. So können identische Anreize bspw. im Lebensmittelhandel andere Effekte bewirken als im Bekleidungshandel (de Wulf et al. 2001). Auch Bolton et al. (2004) weisen auf markt- bzw. branchenspezifische Moderatoren 
der Beziehung zwischen Kundenbindungsinstrumenten und bestimmten Zielgrössen hin. Sie postulieren, dass die aus Konsumentensicht wahrgenommenen Wechselkosten, das wahrgenommene Produktrisiko, die Wettbewerbsintensität und weitere Branchenmerkmale die Wirkung von Anreizen beeinflussen (Bolton et al. 2004, S. 274).

In Hinblick auf beziehungsbezogene Moderatoren ist es bspw. entscheidend, ob die Konsumenten das beworbene Produkt bzw. den Anbieter bereits gut kennen, oder ob es sich um ein relativ unbekanntes Produkt bzw. einen unbekannten Anbieter handelt. Je nach anfänglicher Markenbekanntheit üben Kundenbindungsinstrumente unterschiedliche Wirkungen aus (Roehm et al. 2002). Ebenso unterscheidet sich die Wirkung der Anreize je nachdem wie zufrieden (Keh und Lee 2006) oder loyal (Rust und Verhoef 2005) die Konsumenten sind und wie stark die Beziehung zum Anbieter bereits ist (Gàzquez-Abad et al. 2011).

Auch persönliche Moderatoren sind für die Wirkung der eingesetzten Instrumente entscheidend. Bspw. beeinflusst das Involvement der Konsumenten (Yi und Jeon 2003; Melancon et al. 2011) bzw. das Interesse an bestimmten Warengruppen (Reutterer et al. 2006) die Wirkung von Kundenbindungsanreizen. Darüber hinaus können sich die eingesetzten Instrumente für Konsumenten mit einem hohen bzw. niedrigen Mass an Selbstbestimmung unterschiedlich auswirken (Dholakia 2006).

Aufgrund dieser zusätzlichen Einflussfaktoren (Moderatoren) sind pauschale Aussagen zur Wirkung von Kundenbindungsinstrumenten kaum möglich. Daher haben sich in den vergangenen Jahren zahlreiche Studien mit der Wirkung unterschiedlicher Anreize unter bestimmten Bedingungen beschäftigt. Die wichtigsten empirischen Ergebnisse hierzu finden sich im Folgenden. Hierbei sind nur empirische Studien eingeschlossen, welche die konkrete Wirkung unterschiedlicher Anreize analysieren. Untersuchungen, die sich allgemein mit der Wirkung von Loyalitätsprogrammen auf bestimmte Zielgrössen befassen, ohne konkrete Anreize zu vergleichen (z.B. Lewis 2004; Liu 2007; Liu und Yang 2009; Mägi 2003; Meyer-Waarden 2007; Meyer-Waarden und Benavent 2009; Sharp und Sharp 1997; Taylor und Neslin 2005), finden keine Berücksichtigung, da sie keine Rückschlüsse auf die konkrete Ausgestaltung von Kundenbindungsinstrumenten zulassen.

\section{Empirische Forschungsergebnisse zur Wirkung von Kundenbindungsinstrumenten}

\subsection{Die Wirkung monetärer vs. nicht-monetärer Anreize}

In Hinblick auf den Kundennutzen, der durch die eingesetzten Instrumente entsteht, unterscheidet die Literatur zwischen monetären und nicht-monetären Anreizen. Teilweise finden sich auch die Bezeichnungen ,ökonomische“ und „soziale“ Anreize (Bhattacharya und Bolton 2000; Bolton et al. 2004). Die Unterscheidung geht ursprünglich auf Berry (1995) zurück, der zwischen Level 1-, Level 2- und Level 3-Instrumenten differenziert. Während Level 1-Instrumente meist einen monetären Nutzen bieten, sind Level 2- und Level 3-Instrumente nicht-monetärer Natur. Level 2-Instrumente zielen darauf ab, eine persönliche Bindung zum Kunden herzustellen. Level 3-Instrumente gehen noch einen Schritt weiter, indem sie dem Kunden eine 
individuelle Lösung für ein ganz bestimmtes Problem bieten. Sie repräsentieren die stärkste Form von Kundenbindungsinstrumenten und bergen das höchste Kundenbindungspotenzial (Berry 1995, S. 240f). Kumar und Shah (2004) nehmen eine ähnliche Unterteilung vor und unterscheiden ,tier 1 rewards“ und „tier 2 rewards“. Während erstere monetär sind und direkt auf eine Verhaltensänderung abzielen, sind letztere nicht-monetär und zielen darauf ab, die Einstellung gegenüber dem Anbieter zu verbessern (Kumar und Shah 2004, S. 322-325).

Fragt man die Kunden direkt nach ihrer Präferenz für monetäre und nichtmonetäre Anreize, so schneiden monetäre Anreize besser ab als nicht-monetäre Anreize (Müller 2006, S. 101). Diese subjektive Präferenz schlägt sich jedoch nicht immer in verbesserten Einstellungen gegenüber dem Anbieter und einer höheren Kundenbindung nieder. Daher kommen Studien, welche die Wirkung monetärer und nicht-monetärer Anreize miteinander vergleichen, zu uneinheitlichen Ergebnissen. Verhoef (2003) analysierte im Rahmen einer Studie bei einem Versicherungsanbieter u.a. die Wirkung monetärer Anreize und nicht-monetärer Anreize auf das Kaufverhalten (Share of Wallet). Für beide Instrumente wies er positive Effekte nach (Verhoef 2003, S. 38-40). Auch Helm und Ludl (2005) verglichen die Wirkung monetärer und nicht-monetärer Anreize auf unterschiedliche Einstellungskonstrukte ( $\mathrm{Zu}$ friedenheit, psychologische und ökonomische Wechselkosten, Attraktivität der Alternativen). Ein Vorher-Nachher-Experiment zeigte, dass beide Anreize positive Effekte auf die Zufriedenheit und die ökonomischen Wechselkosten sowie negative Effekte auf die wahrgenommene Attraktivität der Alternativen ausüben. Die psychologischen Wechselkosten wurden allerdings nur von den nicht-monetären Anreizen positiv beeinflusst (Helm und Ludl 2005, S. 1153, 1157). Die Autoren plädieren daher für einen stärkeren Einsatz nicht-monetärer Anreize, da diese durchwegs die stärksten positiven Effekte auf die betrachteten abhängigen Variablen ausüben (Helm und Ludl 2005, S. 1157). Noch differenziertere Ergebnisse zeigt eine Studie von Rust und Verhoef (2005), welche die Wirkung monetärer und nicht-monetärer Anreize auf das Kaufverhalten untersuchte (Rust und Verhoef 2005, S. 479). Mithilfe eines Feldexperimentes in der Versicherungsbranche analysierten sie den Einfluss dieser Instrumente auf den Unternehmensgewinn. Als Moderator berücksichtigten sie die anfängliche Loyalität der Konsumenten. Für loyale Konsumenten beeinflussten nicht-monetäre Anreize das Kaufverhalten stärker positiv als monetäre Anreize (Rust und Verhoef 2005, S. 482-486). Für illoyale Konsumenten hatten hingegen monetäre Anreize einen stärkeren Einfluss als nicht-monetäre. Die Autoren folgerten daher, dass Unternehmen nicht-monetäre Belohnungen zur Kundenbindung und monetäre Belohnungen zur Kundenakquisition einsetzen sollten (Rust und Verhoef 2005, S. 487).

Puchner (2011) verglich die Wirkung monetärer und nicht-monetärer Anreize auf die Einstellungen (wahrgenommene Beziehungsinvestitionen) und das Kaufverhalten (Flugbuchungen) von Kunden einer Airline. Für beide Zielgrössen zeigten sich in dieser Untersuchung positive Effekte von monetären und nicht-monetären Anreizen. Hinsichtlich der Effektivität unterschieden sich die beiden Instrumente nicht (Puchner 2011, S. 221). Zudem erzielten beide Anreize eine stärkere Wirkung für Langzeitkunden als für relativ neue Kunden (Puchner 2011, S. 240-242).

Auch Melancon et al. (2011) verglichen mithilfe von zwei Experimenten die Wirkung monetärer und nicht-monetärer Anreize auf die Einstellungen (,,affective commitment“ und „,continuance commitment“) von Konsumenten. Während ,,affective 
commitment“ die emotionale Bindung zum Unternehmen meint, beschreibt ,,continuance commitment" die Notwendigkeit in einer Beziehung zu bleiben, da anderenfalls zeitliche oder monetäre Investitionen verloren gehen (,Lock-In-Effekt"). Das ,,affective commitment“" geht deshalb mit einer starken intrinsischen Motivation, das ,, continuance commitment" hingegen mit einer starken extrinsischen Motivation der Konsumenten einher (Melancon et al. 2011, S. 343). Die Autoren differenzierten die Anreize zudem nach dem Kontrollgrad, d.h. ob das Einlösen an bestimmte Bedingungen oder Regeln geknüpft ist (z.B. Kleingedrucktes, Gültigkeitsdatum eines Gutscheins). Sie fanden heraus, dass nicht-monetäre Anreize das ,affective commitment“, also die emotionale Bindung zum Unternehmen, positiv beeinflussen, während monetäre Anreize keinen Einfluss darauf haben. Im Gegensatz dazu beeinflussen monetäre Anreize das ,continuance commitment“ positiv, wohingegen nicht-monetäre Anreize hierauf keinen Effekt haben. Knüpfte das Unternehmen das Einlösen der Belohnung an bestimmte Bedingungen, so ergaben sich sowohl für monetäre als auch für nichtmonetäre Anreize negative Effekte auf die beiden abhängigen Variablen. Das heisst, die ursprünglich positiven Effekte kehrten sich um (Melancon et al. 2011, S. 352357). Die Studie zeigt, dass monetäre Belohnungen kurzfristig orientiert sind und die extrinsische Motivation der Konsumenten beeinflussen, während nicht-monetäre Anreize langfristig wirken und die emotionale Bindung zum Unternehmen stärken. Wenn Anbieter Belohnungen an Bedingungen knüpfen, können sich positive Effekte jedoch ins Negative umkehren.

Eine weitere Differenzierung monetärer Anreize nahmen Hennig-Thurau und Paul (2007) vor. Sie testeten im Rahmen eines Online-Experimentes die Wirkung vier unterschiedlicher monetärer Anreize auf Einstellungen (intrinsische und extrinsische Motivation) und Verhaltensintentionen (Wiederkaufabsicht und Wechselabsicht) von Restaurantbesuchern. Wie die Studie von Melancon et al. (2011) zeigte auch diese Untersuchung, dass alle monetären Anreize die extrinsische Motivation erhöhen. Sie senken gleichzeitig die wahrgenommene Selbstbestimmung und beeinflussen die Loyalität langfristig negativ (Hennig-Thurau und Paul 2007). Für die vier unterschiedlichen monetären Anreize zeigten sich keine signifikanten Unterschiede. Die Autoren folgerten daher, dass nicht-monetäre Anreize generell überlegen sind (Hennig-Thurau und Paul 2007, S. 170-173).

Zusammenfassend ist festzuhalten, dass monetäre Anreize unter bestimmten Bedingungen zwar in der Lage sind, das Kaufverhalten zu stimulieren. Aufgrund ihrer negativen Langzeiteffekte auf die intrinsische Motivation sollten sie jedoch sparsam eingesetzt und auf bestimmte Zielgruppen (z.B. illoyale Kunden) abgestimmt werden.

\subsection{Die Wirkung materieller vs. immaterieller Anreize}

Neben der Unterscheidung in monetäre und nicht-monetäre Anreize findet sich in der wissenschaftlichen Literatur auch eine Einteilung anhand der Tangibilität der Kundenbindungsinstrumente (Christy et al. 1996, S. 182; de Wulf et al. 2001; Roehm et al. 2002, S. 203). Hierbei ist zwischen materiellen und nicht-materiellen Anreizen zu differenzieren. Kumar und Reinartz sprechen von ,hard rewards“ und „soft rewards“ (Kumar und Reinartz 2006, S. 173). Während materielle Anreize 
direkt greifbar sind (z.B. Werbegeschenke), stiften nicht-materielle Anreize (z.B. Website, Kundenclub, Event) einen indirekten und abstrakten Mehrwert. Bei nichtmateriellen Anreizen entsteht der Kundennutzen bspw. durch spezielle Informationen oder ein Gefühl der Anerkennung (Roehm et al. 2002, S. 203). Monetäre Anreize, wie Coupons und Gutscheine, ordnet die wissenschaftliche Literatur überwiegend den materiellen Anreizen zu, da sie meist in materielle Produkte eingetauscht werden (Christy et al. 1996, S. 182f; de Wulf et al. 2001; O'Malley und Tyann 2000).

De Wulf et al. (2001) untersuchten mit Hilfe einer Kundenbefragung, ob sich der Einsatz von Kundenbindungsinstrumenten auf die Einstellungen der Konsumenten (wahrgenommene Beziehungsinvestitionen des Anbieters) auswirkt. Die Autoren unterschieden immaterielle Anreize (Direkt-Mailings, bevorzugte Behandlung durch den Anbieter, persönliche Ansprache) und materielle Anreize. In drei Ländern befragten sie Kunden von Lebensmittel- sowie Bekleidungshändlern. In beiden Branchen wirkten sich sowohl materielle als auch immaterielle Anreize positiv auf die Einstellungen aus. Allerdings variierten die Ergebnisse sowohl über die beiden Branchen als auch über die drei Länder, so dass kein eindeutiges Muster erkennbar ist (de Wulf et al. 2001, S. 39-45). Diesbezüglich liefern jedoch Roehm et al. (2002) differenziertere Empfehlungen. Sie untersuchten die Wirkung unterschiedlicher Anreize auf die Einstellungen (Markenwahrnehmung) und das Kaufverhalten (Produktwahl) in der Konsumgüterindustrie (Getränke). Mithilfe von Experimenten verglichen sie in mehreren Studien materielle vs. immaterielle sowie anbieterbezogene vs. nichtanbieterbezogene Anreize. Als Moderator betrachteten sie die anfängliche Markenbekanntheit des Produkts beim Konsumenten. Sie fanden heraus, dass insbesondere nicht-materielle, anbieterbezogene Anreize die Markenwahrnehmung und die Produktwahl positiv beeinflussen. Dies gilt allerdings nur für Konsumenten, welche eine Marke vorher noch nicht gut kannten (niedrige anfängliche Markenbekanntheit). Materielle Anreize (anbieterbezogen und nicht-anbieterbezogen) senkten die Loyalität hingegen langfristig, da die positive Einstellung gegenüber der Marke durch eine positive Einstellung gegenüber der Belohnung ersetzt wurde und mit Wegnahme der Belohnung nicht mehr vorhanden war. Besonders stark war dieser negative Effekt für Konsumenten, welche die Marke vorher gut kannten (Roehm et al. 2002, S. 404-410). Die Untersuchung zeigt, dass materielle Anreize insbesondere für Konsumenten, die mit einer Marke vertraut sind, langfristig negative Effekte haben können.

Insgesamt zeigen bestehende Studien, dass immaterielle Anreize den materiellen tendenziell überlegen sind, da sie weniger mit potenziellen negativen Langzeiteffekten behaftet sind. Insbesondere für Kunden, die loyal sind oder eine Marke bereits gut kennen, gefährden materielle Anreize die Loyalität. Auf diesem Feld besteht noch grosser Forschungsbedarf und die Wirkung materieller vs. nicht-materieller Anreize sowie weiterer Moderatoren bedarf einer differenzierteren Betrachtung.

\subsection{Die Wirkung anbieterbezogener vs. nicht-anbieterbezogener Anreize}

Während nicht-anbieterbezogene Anreize das Produkt oder die Kernleistung eines Unternehmen nicht in den Vordergrund stellen, verfolgen anbieterbezogene Anreize das Ziel, direkt das Produkt bzw. den Anbieter zu bewerben und dessen Mehrwert herauszustellen (Dowling und Uncles 1997, S. 76; Keh und Lee 2006, S. 128; 
Kumar und Reinartz 2006, S. 173; Roehm et al. 2002, S. 203; Yi und Jeon 2003, S. 230). Roehm et al. sprechen in diesem Zusammenhang von ,cue-compatible“ (anbieterbezogenen) und ,cue-incompatible“ (nicht-anbieterbezogenen) Anreizen (Roehm et al. 2002, S. 203). Bonusmeilen, welche Kunden als Belohnung für ihre Treue bei zahlreichen Airlines erhalten, stellen typische anbieterbezogene Anreize dar. Nicht-anbieterbezogene Anreize sind hingegen alle anderen Belohnungen, die keine direkten Assoziationen in Bezug auf die Kernleistung des Unternehmens hervorrufen.

Eine Sonderform bilden Loyalitätsprogramme in Kooperation mehrerer Anbieter (Evanschitzky et al. 2012; Hartmann 2001; Müller 2006, S. 86f). Hier weisen die Kundenbindungsinstrumente entweder einen Bezug zum Anbieter (z.B. Meilensammeln durch Fliegen), einen Bezug zu einem der Partnerunternehmen (z.B. Meilen sammeln durch Bezahlung mit Kreditkarte) oder keinen Bezug zu einem der teilnehmenden Unternehmen auf (Lemon und von Wangenheim 2009).

Roehm et al. (2002) (vgl. Abschn. 4.2) vergleichen die Wirkung anbieterbezogener und nicht-anbieterbezogener Anreize auf die Einstellungen (Markenwahrnehmung) und das Kaufverhalten (Produktwahl). Die Autoren unterschieden die Anreize zudem nach ihrer Tangibilität in materielle und immaterielle Anreize. Roehm et al. (2002) bestätigen insbesondere die positive Wirkung anbieterbezogener Anreize auf die Markenwahrnehmung und die Produktwahl. Der Einfluss nicht-anbieterbezogener Anreize war hingegen schwächer. Anbieterbezogene Anreize wirkten am stärksten, wenn sie in immaterieller Form (z.B. Newsletter zum Produkt) angeboten wurden (Roehm et al. 2002, S. 404-410). Auch Tietje (2002) verglich die Wirkung anbieterund nicht-anbieterbezogener Anreize auf die Einstellungen (Zufriedenheit und Wiederkaufabsicht) von Konsumenten. Zudem unterschied er zwischen sofortigen und verzögerten Anreizen. Die Studie zeigt, dass sich anbieterbezogene sofortige Anreize stärker auf die Einstellungen auswirken als anbieterbezogene verzögerte Anreize. Gleichzeitig hatten nicht-anbieterbezogene verzögerte Anreize einen stärkeren Effekt als nicht-anbieterbezogene sofortige Anreize. Demnach gilt es Anreize mit Bezug zum Anbieter eher als sofortige Anreize zu gestalten, wohingegen Anreize ohne Anbieterbezug besser als verzögerte Anreize wirken. $\mathrm{Zu}$ ähnlichen Ergebnissen kamen Keh und Lee (2006): Sie unterschieden die Anreize ebenfalls nach dem Anbieterbezug und betrachteten zusätzlich die Zufriedenheit der Konsumenten. Sie zeigten, dass anbieterbezogene sofortige Anreize nur für unzufriedene Konsumenten überlegen sind, während für zufriedene Konsumenten anbieterbezogene verzögerte Anreize überlegen sind. Sie bestätigen damit die Untersuchung von Tietje für unzufriedene Konsumenten. Allerdings waren in dieser Untersuchung nicht-anbieterbezogene Anreize - unabhängig von der zeitlichen Wirkung und der Zufriedenheit der Konsumenten - in keiner Situation überlegen.

Auch Puchner (2011) verglich in seinem Experiment den Effekt anbieter- und nicht-anbieterbezogener Anreize auf die Einstellungen und das Kaufverhalten der Konsumenten (vgl. Abschn. 4.1). Er findet für beide Anreize einen positiven Effekt, wobei sich die beiden Instrumente in ihrer Wirkungsstärke nicht unterscheiden. Die Beziehungsdauer des Kunden zum Anbieter moderiert (verstärkt) die Wirkung der beiden Anreizarten (Puchner 2011, S. 239-242).

In Bezug auf Anreize im Rahmen von firmenübergreifenden Loyalitätsprogrammen zeigt Müller (2006, S. 101), dass Konsumenten diese gegenüber nicht- 
kooperativen Programmen präferieren. Lemon und von Wangenheim (2009) bestätigen, dass firmenübergreifende Anreize Konsumenten zum „Cross-Buying“, d.h. zum Einkauf bei einem oder mehreren Partnerunternehmen, motivieren. Das Kaufverhalten wird besonders positiv beeinflusst, wenn die Partnerunternehmen und die jeweiligen Anreize gut zueinander passen (hoher Fit) und die Anreize somit einen gewissen Anbieterbezug aufweisen (vgl. auch Hartmann 2001, S. 13). Gleichzeitig erreichen Unternehmen mit derartigen Loyalitätsprogrammen jedoch häufig eine Kundenbindung an das Programm und nicht an das jeweilige Unternehmen (Evanschitzky et al. 2012; Tomczak et al. 2005, S. 395). Der einzelne Anbieter kann durch solche Programme die Loyalität seiner Kunden nicht immer positiv beeinflussen, da die Konsumenten auch bei einem beliebigen anderen Anbieter aus dem Programm einkaufen können (Sharp und Sharp 1997, S. 484).

Aus Basis der aufgezeigten Studien ist zusammenzufassen, dass anbieterbezogene Anreize den nicht-anbieterbezogenen generell überlegen sind. Werden die Anreize in verzögerter Form eingesetzt, kann auch die Wahl nicht-anbieterbezogener Anreize sinnvoll sein. Für firmenübergreifende Loyalitätsprogramme ist es wichtig, dass die Unternehmen und Anreize gut zueinander passen und somit ebenfalls ein gewisser Anbieterbezug besteht.

\subsection{Die Wirkung direkter vs. indirekter Anreize}

Eine weitere Kategorisierung von Kundenbindungsinstrumenten unterscheidet zwischen direkten und indirekten Anreizen (Dowling und Uncles 1997; Keh und Lee 2006; Rothschild und Gaidis 1981). Rothschild und Gaidis (1981) sprechen in diesem Zusammenhang von ,primären“ und „sekundären“ Belohnungen. Während direkte (primäre) Anreize - vergleichbar mit sofortigen Anreizen - einen immanenten Nutzen für den Kunden stiften, sind indirekte (sekundäre) Anreize erst nach dem Einlösen nutzenstiftend. Im Unterschied zu verzögerten Anreizen spielt die zeitliche Komponente bei dieser Systematik jedoch keine Rolle. Beispiele für direkte Anreize sind Geschenke oder besondere Serviceleistungen. Hierbei entsteht ein unmittelbarer Nutzen für den Kunden. Gutscheine, Coupons oder Einladungen zu Events stellen hingegen indirekte Anreize dar, da sie keinen direkten Nutzen stiften, sondern erst nach dem Einlösen nutzenstiftend sind (Keh und Lee 2006, S. 128).

Yi und Jeon (2003) testeten mithilfe eines Experiments die Wirkung direkter und indirekter Anreize auf die Einstellungen (wahrgenommener Wert des Loyalitätsprogramms) und das Kaufverhalten (Loyalität) der Konsumenten. Sie unterschieden zudem zwischen sofortigen und verzögerten Anreizen und betrachteten das Involvement der Konsumenten (hoch vs. niedrig) als Moderator. Die Ergebnisse zeigten, dass bei hohem Involvement direkte Belohnungen einen grösseren Einfluss auf die Einstellungen der Konsumenten ausüben als indirekte. Für niedriges Involvement ergaben sich hingegen keine signifikanten Unterschiede. Direkte Belohnungen sind den indirekten damit generell überlegen und insbesondere für hoch involvierte Kunden bzw. in Märkten mit „High-Involvement-Produkten“ vorzuziehen (Yi und Jeon 2003, S. 236238). 


\subsection{Die Wirkung sofortiger vs. verzögerter Anreize}

Eng verwandt mit der Unterscheidung direkter und indirekter Anreize ist die Unterteilung in sofortige und verzögerte Anreize (Dowling und Uncles 1997, S. 76; Reinartz und Kumar 2000, S. 174; Rothschild und Gaidis 1981, S. 73). Im ersten Fall erhalten Kunden direkt zum Zeitpunkt der Transaktion eine Belohnung (z.B. sofortiger Preisnachlass beim Einkauf). Verzögerte Belohnungen stiften hingegen erst nach einer bestimmten Zeit oder Anzahl von Einkäufen einen Nutzen für den Kunden (Keh und Lee 2006, S. 128; Yi und Jeon 2003, S. 230). Zhang et al. (2000) sprechen auch von ,front-loaded“ (sofortigen) und ,rear-loaded“ (verzögerten) Anreizen (Zhang et al. 2000, S. 348). In die letztgenannte Kategorie fallen bspw. klassische Punkte-Sammelprogramme, wie sie zahlreiche Händler, Airlines und andere Serviceorganisationen anbieten. Erst nach dem Sammeln einer bestimmten Anzahl von Punkten, erhält der Kunde einen Preisnachlass oder eine andere Belohnung. Auch Gutscheine, die erst bei einem Folgekauf eingesetzt werden dürfen, stellen verzögerte Belohnungen dar (Keh und Lee 2006, S. 128).

Zhang et al. (2000) untersuchten die Wirkung sofortiger und verzögerter Anreize auf den Umsatz und Gewinn im Lebensmittelhandel. Sie fanden heraus, dass die Vorteilhaftigkeit der beiden Anreizarten davon abhängt, ob die Konsumenten ein hohes Streben nach Abwechslung (,,variety seeking“), oder aber eine hohe Konstanz (,inertia“) in ihrem Kaufverhalten aufweisen. Im ersten Fall sind verzögerte Anreize profitabler, während im letzten Fall sofortige Anreize vorzuziehen sind. Auch die im vorigen Abschnitt vorgestellte Studie von Yi und Jeon (2003) unterschied neben direkten und indirekten auch sofortige (z.B. sofortiger Preisnachlass) und verzögerte (z.B. Bonuspunkte) Anreize. Für stark involvierte Konsumenten zeigten sich keine Unterschiede zwischen sofortigen und verzögerten Anreizen in Hinblick auf die Beeinflussung der Einstellungen und des Kaufverhaltens. Für schwach involvierte Konsumenten hatten hingegen sofortige Belohnungen einen stärkeren Effekt als nachträgliche. Daher sind sofortige Anreize den verzögerten - insbesondere in Märkten mit niedrigem Involvement - überlegen (Yi und Jeon 2003, S. 236-238). Auch Keh und Lee (vgl. Abschn. 4.3) untersuchten die zeitliche Wirkung von Anreizen. Sie fanden heraus, dass für anfänglich zufriedene Kunden nachträgliche Anreize einen stärkeren Effekt auf die Loyalität haben, während für unzufriedene Konsumenten sofortige Anreize effektiver sind (Keh und Lee 2006). Tietje (2002) (vgl. Abschn. 4.3) zeigte, dass sofortige anbieterbezogene Anreize einen stärkeren positiven Einfluss auf die Zufriedenheit und die Wiederkaufabsicht haben als verzögerte anbieterbezogene Anreize. Hingegen haben verzögerte nicht-anbieterbezogene Anreize eine stärkere positive Wirkung als sofortige nicht-anbieterbezogene Anreize.

In Hinblick auf die zeitliche Wirkung lässt sich aufgrund der bestehenden Literatur somit folgern, dass sofortige Anreize den verzögerten, insbesondere für eher unzufriedene oder schwach involvierte Konsumenten (z.B. Neukunden, Gelegenheitskunden), überlegen sind. Sie sollten zudem einen Anbieterbezug aufweisen. Verzögerte Anreize können für zufriedene, hoch involvierte und nach Abwechslung strebende Kunden eingesetzt werden und müssen keinen Anbieterbezug aufweisen. 


\subsection{Die Wirkung informativer vs. einschränkender Anreize}

Eine weitere Klassifikation von Kundenbindungsinstrumenten unterscheidet zwischen informativen und einschränkenden Anreizen. Während erstere rein informativer Natur für den Kunden sind, kontrollieren letztere das Verhalten der Kunden in einer bestimmten Weise und schränken seine Autonomie ein (z.B. Dholakia 2006; Hennig-Thurau und Paul 2007; Melancon et al. 2011). Insbesondere Belohnungen, die an Restriktionen geknüpft werden, stellen einschränkende Anreize dar, da die Kunden in ihrer Entscheidungsfreiheit berührt werden (Graham 1994; Melancon et al. 2011; Tietje 2002). Derartige Einschränkungen entstehen bspw. durch Bedingungen beim Einlösen der Belohnung. Dies sind z.B. Mengenbegrenzungen, Beschränkungen auf bestimmte Warengruppen oder die Angabe eines Gültigkeitsdatums. Auch wenn der Kunde zunächst eine bestimmte Leistung (z.B. Mindesteinkaufswert) erfüllen muss, um eine Belohnung zu erhalten, stellt dies eine Einschränkung seiner Entscheidungsfreiheit dar (Ryan et al. 1983, S. 738).

Melancon et al. (2011) (vgl. Abschn. 4.1) verglichen den Einfluss informativer und einschränkender Anreize auf das ,,affective commitment“ und das ,continuance commitment“. Die Ergebnisse zeigten, dass einschränkende Anreize sowohl das ,affective commitment“ als auch das ,continuance commitment“ negativ beeinflussen. Selbst die ursprünglich positiven Effekte von monetären und nicht-monetären Anreizen auf die Einstellungen kehrten sich um, sobald die Anreize an Bedingungen geknüpft wurden.

Auch Dholakia (2006) untersuchte die Wirkung von informativen bzw. einschränkenden Anreizen und betrachtete zudem die wahrgenommene Selbstbestimmung von Konsumenten. Als einschränkenden Anreiz verwendete der Autor ErinnerungsCoupons; der informative Anreiz war die Mitgliedschaft in einem speziellen Loyalitätsprogramm (Dholakia 2006, S. 114). Wie bei Melancon et al. (2011) zeigte sich, dass Konsumenten mit einem hohen Mass an Selbstbestimmung ErinnerungsCoupons als Kontrollinstrument wahrnehmen und die Loyalität langfristig sank. Hingegen empfinden Konsumenten mit einem niedrigen Mass an Selbstbestimmung die Coupons nicht als Kontrolle, ihre Loyalität stieg daher an. Der informative Anreiz wirkte sich für Konsumenten mit einem hohen Mass an Selbstbestimmung positiv auf die Loyalität aus, während sich für Konsumenten mit einem niedrigen Mass an Selbstbestimmung kein Effekt ergab. Nach dieser Studie sollte der Einsatz kontrollierender oder einschränkender Anreize nur für Konsumenten erfolgen, die ein niedriges Mass an Selbstbestimmung aufweisen. Für alle anderen Konsumenten ergeben sich negative Effekte. Erhalten bspw. Konsumenten mit einem hohen Mass an Selbstbestimmung einen Erinnerungs-Coupon, sind nicht nur verlorene Investitionen die Folge; es ergeben sich langfristig sogar schädliche Auswirkungen auf die Loyalität (Dholakia 2006).

Aufgrund der existierenden Literatur lässt sich zusammenfassen, dass Anreize eher informativ ausgestaltet und nicht an Bedingungen geknüpft werden sollten. Einzig für Konsumenten mit einem niedrigen Mass an Selbstbestimmung zeigten einschränkende Anreize positive Effekte. Da Konsumenten ihre Kaufentscheidungen in der Regel jedoch aus freien Stücken treffen und damit generell ein hohes Mass an Selbstbestimmung aufweisen, sind informative Anreize meist überlegen. 


\subsection{Die Wirkung beziehungs- vs. verhaltensorientierter Anreize}

Bliemel und Eggert (1998) unterscheiden danach, ob die Kundenbindung auf positiven Einstellungen des Kunden beruht oder lediglich durch das entsprechende Kaufverhalten gekennzeichnet ist. Sie unterscheiden daher zwischen „Gebundenheit“ und „Verbundenheit“. Während gebundene Kunden aufgrund bestimmter Wechselbarrieren loyal zu einem Anbieter sind, verhalten sich verbundene Kunden aufgrund ihrer positiven Einstellung gegenüber dem Anbieter loyal. Auf Verbundenheit ausgerichtete kundenbindende Aktivitäten zielen entsprechend auf eine Verbesserung der Einstellungen (z.B. Zufriedenheit, Commtiment, Vertrauen) ab. Sie sollen die emotionale Beziehung zum Kunden stärken. Kundenbindungsinstrumente, welche die Gebundenheit fördern, beeinflussen hingegen, z.B. durch den Aufbau von Wechselbarrieren, direkt das Kaufverhalten (Bliemel und Eggert 1998, S. 38-43; Bolton et al. 2004, S. 278; Bruhn 2009, S. 86; Dick und Basu 1994; McDonald 1998). Die wissenschaftliche Literatur unterscheidet daher zwischen beziehungsorientierten und verhaltensorientierten Kundenbindungsinstrumenten. Gàzquez-Abad et al. (2011, S. 168f) sprechen auch von ,,promotional“ bzw. „,call-to-action“ sowie ,,relational“ bzw. ,retailimage enhancement" orientierten Anreizen. Kumar und Shah (2004, S. 322-324) bezeichnen ,tier 1 rewards“ als verhaltensorientierte und ,tier 2 rewards“ als beziehungsorientierte Anreize (vgl. Abschn. 4.1). Da auch beziehungsorientierte Anreize ein bestimmtes Verhalten der Kunden (Aufrechthalten der Beziehung zum Anbieter) beschreiben, scheint die Unterteilung auf den ersten Blick nicht ganz trennscharf. Entscheidend ist hierbei, dass die verhaltensorientierten Anreize direkt auf eine Verhaltensreaktion der Konsumenten abzielen, während die beziehungsorientierten Anreize in einem ersten Schritt die Wahrnehmungen und Einstellungen der Konsumenten verbessern sollen. Erst im zweiten Schritt geht es hier um eine Verhaltensreaktion aufgrund der vorherigen Einstellungsänderung. Man könnte folglich auch von „einstellungs-“ und ,,verhaltensorientierten“ Anreizen sprechen. Generell weisen monetäre Anreize einen starken Verhaltensbezug auf, da sie den Kunden direkt zu einem bestimmten Verhalten auffordern. Nicht-monetäre Anreize sind hingegen eher beziehungsorientiert; der Aufforderungscharakter ist weniger stark (Bhattacharya und Bolton 2000, S. 343).

Gàzquez-Abad et al. (2011) testeten die kurz- und langfristigen Wirkungen beziehungs- und verhaltensorientierter Direkt-Mailings auf das Kaufverhalten im Bekleidungshandel. Während verhaltensorientierte Mailings eine konkrete Aufforderung (,call-to-action“) enthielten, waren beziehungsorientierte Mailings rein informativ ausgestaltet. Als Moderator bezogen sie die ursprüngliche Beziehungsstärke zwischen Anbieter und Kunden ein. Die Ergebnisse zeigten, dass kurzfristig sowohl beziehungsorientierte als auch verhaltensorientierte Mailings einen positiven Effekt auf das Kaufverhalten ausüben, wobei der positive Effekt beziehungsorientierter Mailings stärker ist. Auf lange Sicht hatten verhaltensorientierte Mailings einen negativen Effekt auf das Kaufverhalten, während beziehungsorientierte Mailings das Kaufverhalten nach wie vor positiv beeinflussten. Der negative Effekt der verhaltensorientierten Mailings überstieg sogar den positiven Effekt der beziehungsorientierten Mailings (Gàzquez-Abad et al. 2011, S. 175). Die Beziehungsstärke moderierte die Zusammenhänge: Sie schwächte den negativen Langzeiteffekt der verhaltensorientierten 
Mailings ab; d.h. je stärker die Beziehung zwischen dem Händler und dem Kunden ursprünglich war, desto schwächer war der negative Langzeiteffekt der verhaltensorientierten Mailings auf das Kaufverhalten. Allerdings senkte eine hohe Beziehungsstärke auch den positiven kurzfristigen Effekt der verhaltensorientierten Mailings. Auf den Effekt der beziehungsorientierten Mailings übte die Beziehungsstärke hingegen keinen moderierenden Einfluss aus (Gàzquez-Abad et al. 2011, S. 176f). Die Autoren folgerten daher, dass Anreize generell eher beziehungsorientiert auszugestalten sind. Beziehungsorientierte Anreize üben sowohl kurz- als auch langfristig und unabhängig von der Beziehungsstärke positive Effekte auf das Kaufverhalten aus. Verhaltensorientierte Mailings, welche die Kunden z.B. durch Preisaktionen direkt zum Kauf aufrufen, schaden dem Händlerimage hingegen auf lange Sicht (GàzquezAbad et al. 2011, S. 177-179).

\subsection{Die Wirkung von unterschiedlich kommunizierten Anreizen}

Eine weitere Unterteilung der Kundenbindungsinstrumente unterscheidet nach der Art und Weise, wie sie dem Kunden kommuniziert werden (z.B. Godfrey et al. 2011; Reutterer et al. 2006, S. 52). Diese Unterscheidung ist insbesondere in Hinblick auf digitalisierbare Anreize relevant. Dies sind bspw. Coupons oder Newsletter, die ein Anbieter per Post, per E-Mail oder über mobile Kommunikation versenden kann (Godfrey et al. 2011, S. 96). In Abhängigkeit vom Kommunikationskanal können Kundenbindungsinstrumente unterschiedliche Wirkungen auf Kundenseite hervorrufen und gleichzeitig auf Händlerseite unterschiedlich hohe Kosten verursachen (Reutterer et al. 2006).

Godfrey et al. (2011) verglichen in ihrer Studie unterschiedliche Kommunikationskanäle von Kundenbindungsinstrumenten. Hierfür testeten sie inwieweit sich die Anzahl beziehungsorientierter Kontaktaufnahmen durch einen Autohändler über unterschiedliche Kanäle (Telefon, E-Mail, Brief) auf das Kaufverhalten (Einkaufshäufigkeit und Ausgaben) auswirkt. Generell hatten Briefe den stärksten positiven Einfluss, gefolgt von E-Mails und Telefonkontakten. Die Autoren fanden zudem heraus, dass für jede der drei Kommunikationsarten eine optimale Anzahl an Kontakten existiert. Ab diesem Idealpunkt führt eine Erhöhung zu negativen Effekten auf das Kaufverhalten. In einem Zeitraum von 39 Monaten wirken sich ca. drei Telefonkontakte, 3-4 E-Mail-Kontakte und 9-10 Briefe optimal auf das Kaufverhalten aus (vgl. auch Müller 2006, S. 101). Werden E-Mails und telefonische Kontakte bzw. Briefe zusammen eingesetzt, ergeben sich allerdings negative Interaktionseffekte, d.h., die optimale Anzahl der Kontaktaufnahmen sinkt entsprechend. Auch Reutterer et al. (2006) verglichen unterschiedliche Kommunikationskanäle im Rahmen eines FeldExperiments bei einem DIY-Händler. Sie sendeten Direkt-Mailings via Post bzw. E-Mail an drei Kundensegmente mit unterschiedlichem Produktinteresse (,Fliesenkäufer“, „Gartenartikelkäufer“ und eine Zufallsstichprobe). Die Mailings und Briefe behandelten unterschiedliche Produktthemen. Anschliessend analysierten sie das Kaufverhalten der drei Segmente innerhalb eines Jahres nach Versenden der Anreize. Die Autoren fanden keinen Effekt der Kommunikationsart (E-Mail oder Brief) auf das Kaufverhalten. Aufgrund der niedrigeren Kosten plädierten die Autoren daher dafür, E-Mails generell vorzuziehen (Reutterer et al. 2006). 
Aufgrund bestehender Studien lässt sich folglich keine überlegene Kommunikationsart von Kundenbindungsinstrumenten identifizieren. Allerdings unterscheidet sich je nach Kommunikationskanal die optimale Anzahl der Kontakte. Während Telefonsowie E-Mail-Kontaktaufnahmen eher sparsam erfolgen sollten, dürfen Briefe in kürzeren Abständen eingesetzt werden.

Tabelle 3 fasst die Ergebnisse der beschriebenen Studien zusammen und gibt einen Überblick.

Nachdem die empirischen Erkenntnisse zur Wirkung von Kundenbindungsinstrumenten dargestellt wurden, diskutiert der folgende Abschnitt die Implikationen, die sich für die praktische Ausgestaltung der Anreize ergeben.

\section{Diskussion zum Einsatz von Kundenbindungsinstrumenten aus praktischer Sicht}

\subsection{Ausgestaltung der Instrumente}

Die Zusammenfassung der bestehenden Studien zeigt, dass der Einsatz von Kundenbindungsinstrumenten stets gut abzuwägen ist, da diese unter bestimmten Bedingungen wirkungslos sind, oder sogar negative Effekte auf die Einstellungen und das Kaufverhalten der Konsumenten ausüben können (z.B. Dholakia 2006; Keh und Lee 2006; Hennig-Thurau und Paul 2007; Gàzquez-Abad et al. 2011). Generell besteht die Gefahr, ein ursprünglich intrinsisches Verhalten zu instrumentalisieren und so die intrinsische Motivation zu untergraben. Selbst wenn Kunden nur aus reiner Gewohnheit immer wieder bei einem bestimmten Anbieter einkaufen, ohne in hohem Mass intrinsisch motiviert zu sein, besteht die Gefahr, diese Gewohnheit durch die Instrumentalisierung mit Anreizen zu durchbrechen (Henderson et al. 2011). Die empirischen Studien haben gezeigt, dass gesamtheitlich betrachtet sowohl kurz- als auch langfristig nicht-monetäre, immaterielle, anbieterbezogene, direkte, informative und beziehungsorientierte Anreize überlegen sind. Insbesondere monetäre sowie materielle Anreize, welche starke Formen von extrinsischen Anreizen darstellen (Melancon et al. 2011; Hennig-Thurau und Paul 2007; Roehm et al. 2002), eignen sich eher als Instrument zur Akquisition von Neukunden als zur Kundenbindung (Rust und Verhoef 2005, S. 487). Auch andere verhaltensorientierte oder einschränkende Anreize beeinflussen die Einstellungen und das Kaufverhalten treuer Kunden gegenüber einem Anbieter negativ (Gàzquez-Abad et al. 2011; Melancon et al. 2011). Daher ist bei der Ausgestaltung darauf zu achten, die Entscheidungsfreiheit der Konsumenten so wenig wie möglich einzuschränken (Dholakia 2006; Melancon et al. 2011) und Anreize nicht an strenge Bedingungen zu knüpfen (z.B. Mindesteinkaufswert, Gültigkeitsdatum). Keinesfalls sollten Anbieter diese Anreizarten für Kunden einsetzen, die - aus Zufriedenheit oder Gewohnheit - ohnehin treu sind.

\subsection{Verhindern von Cherry-Picking Verhalten}

Eine Gefahr, die insbesondere mit dem Einsatz monetärer, materieller und verhaltensorientierter Anreize einhergeht, besteht darin, Kunden zu einem Cherry Picking Ver- 


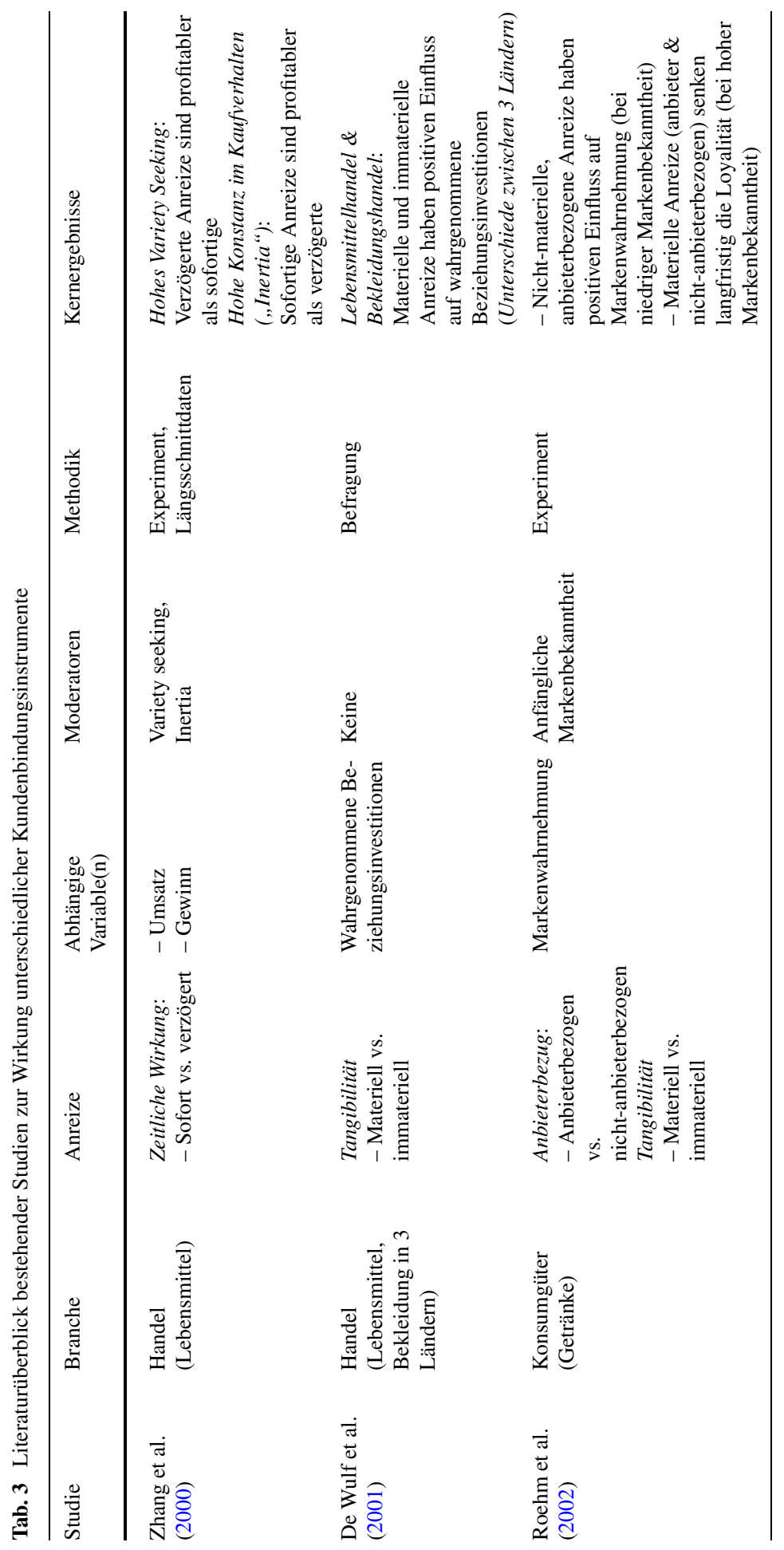




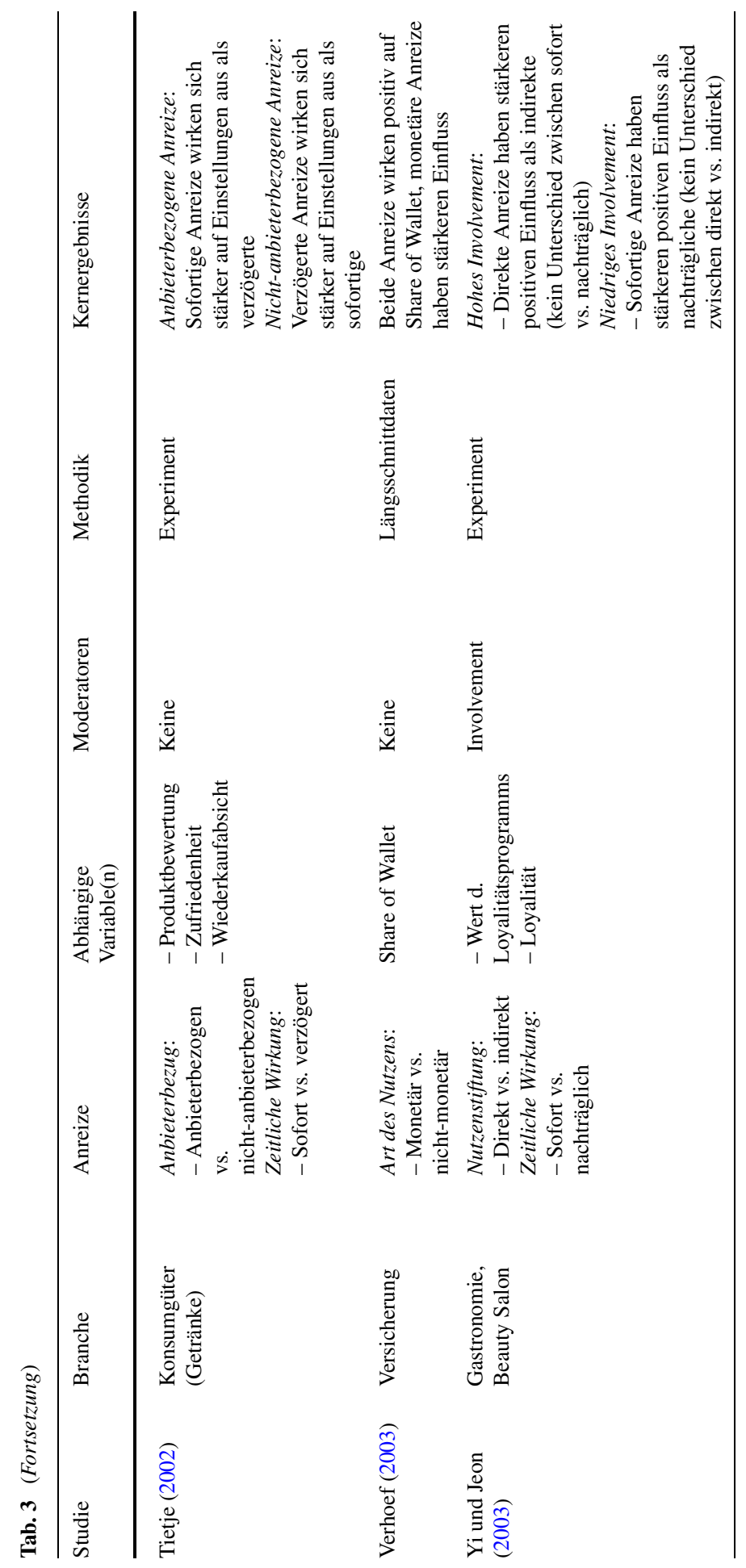




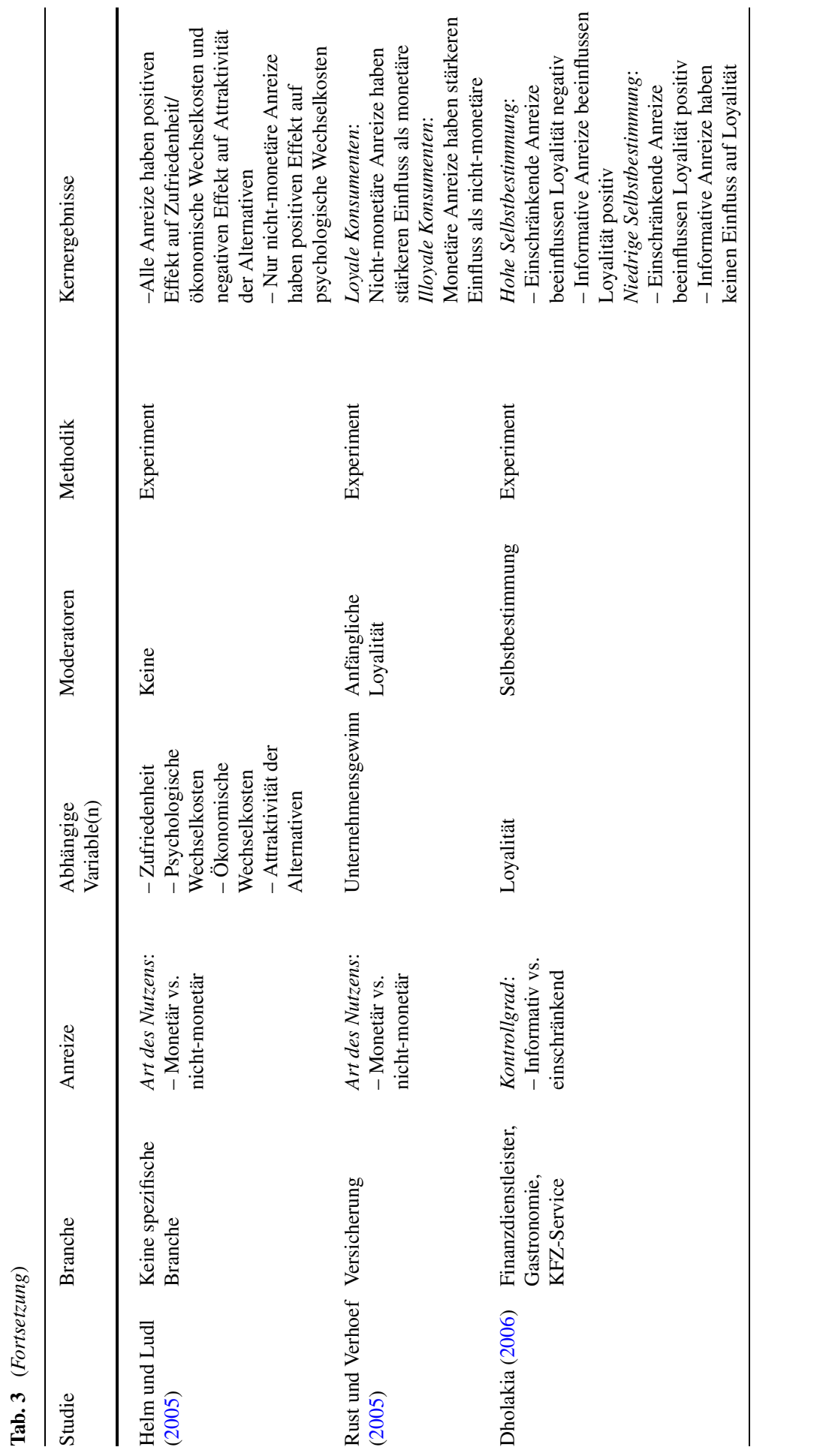




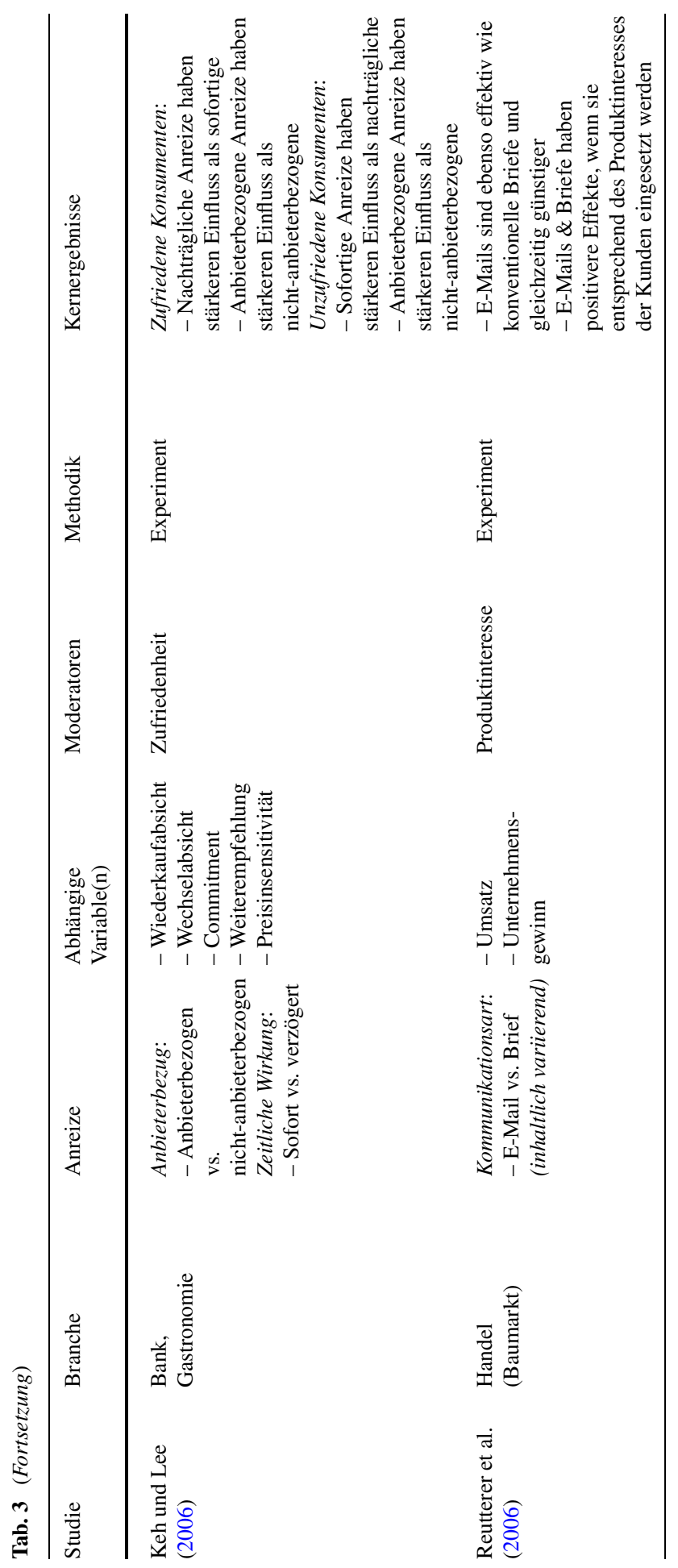




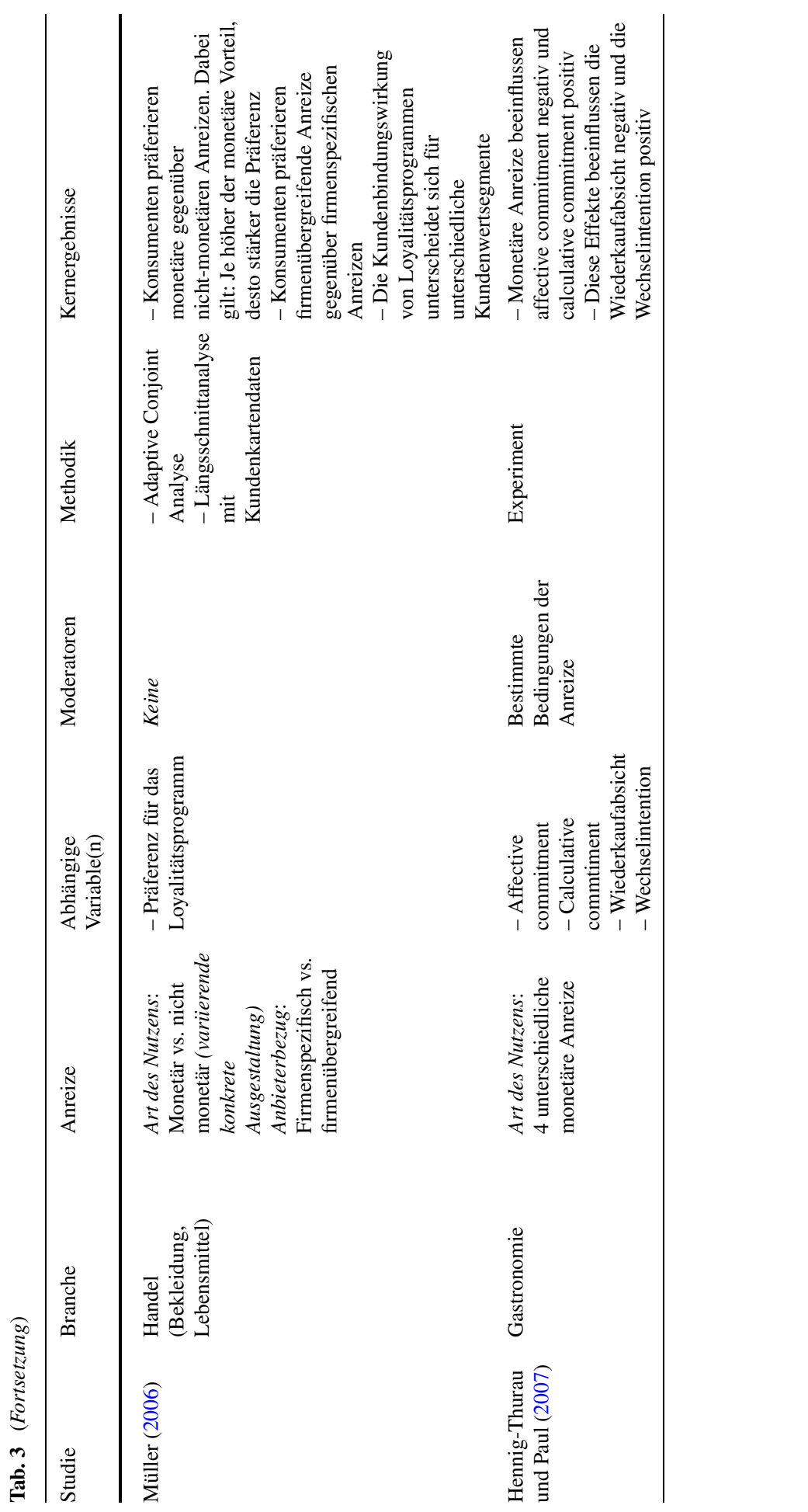




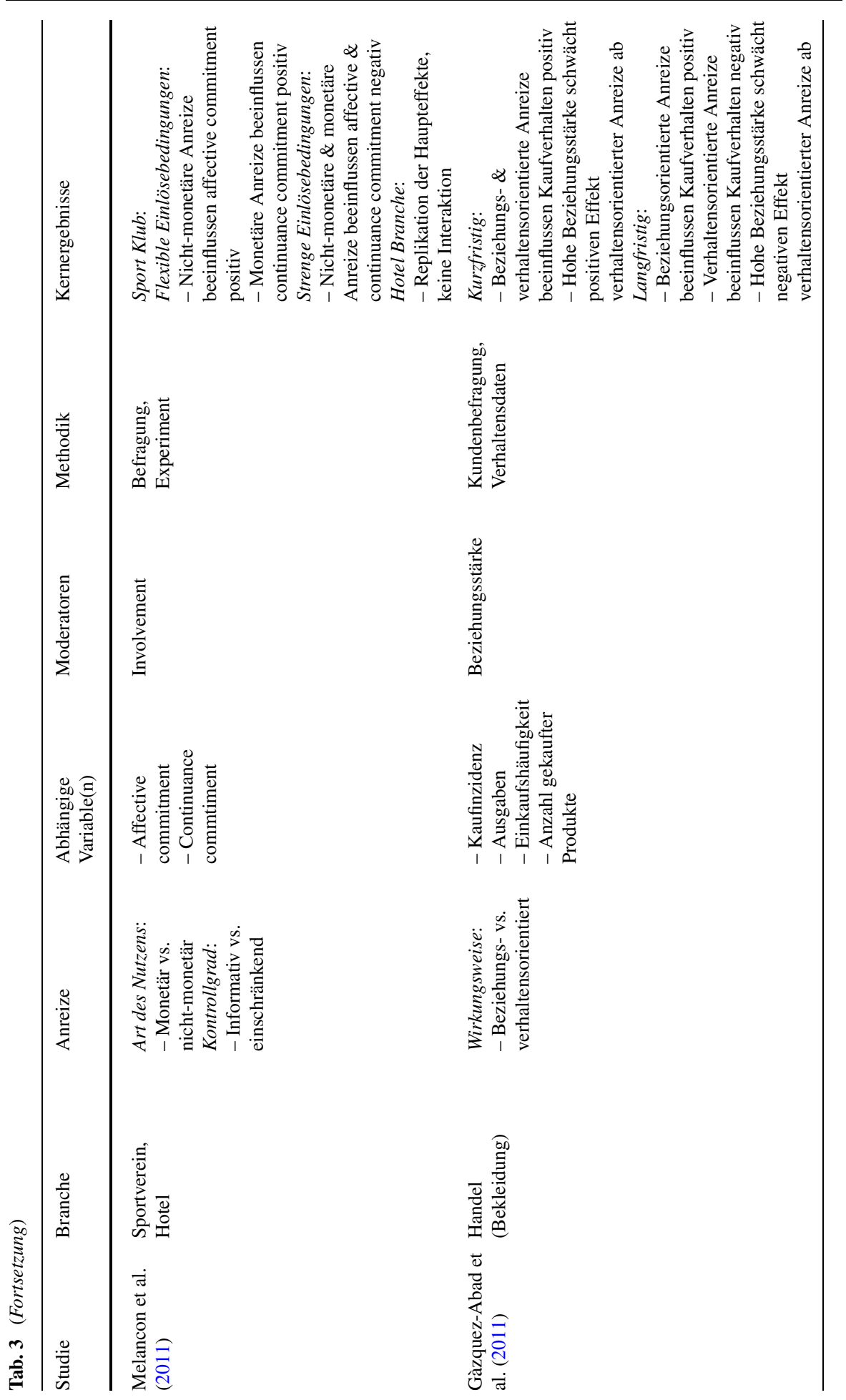




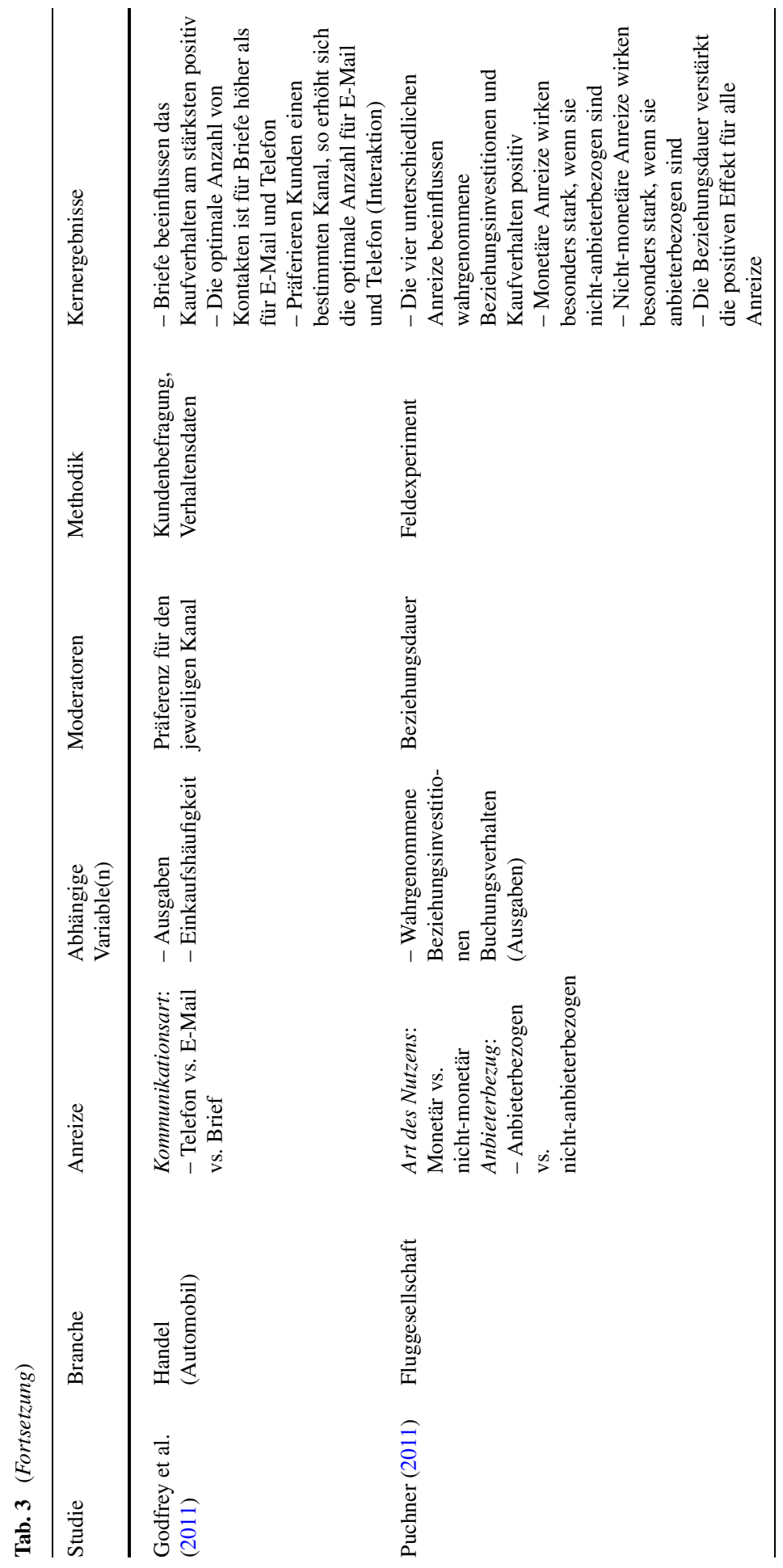


halten zu erziehen. Da diese Anreizarten leicht imitierbar sind, setzen unterschiedliche Händler vergleichbare Anreize im Rahmen ihres Loyalitätsprogramms ein (Berry 1995, S. 240). Viele Konsumenten nehmen an mehreren Loyalitätsprogrammen teil und nutzen die jeweiligen Anreize unterschiedlicher Anbieter aus. Selbst wenn der Umsatz kurzfristig ansteigt, generiert ein solches Verhalten für Handelsunternehmen insgesamt meist keine Mehrumsätze und wirkt sich oftmals letztlich sogar negativ auf den Umsatz aus, da es preisachtsame Kunden anzieht und Stammkunden zur Preisachtsamkeit erzieht. Gleichzeitig kaufen bestehende Kunden, welche ohnehin den Anbieter gewählt hätten, günstiger ein (Henderson et al. 2011; Kumar und Shah 2004; Lal und Bell 2003; Leenheer et al. 2007; Meyer-Waarden 2007; Reutterer et al. 2006). Um ein solches Cherry-Picking Verhalten zu vermeiden, sind v.a. monetäre, materielle und verhaltensorientierte Anreize sparsam und nicht aktionistisch als Reaktion auf Massnahmen von Wettbewerbern anzuwenden.

\subsection{Förderung von Verbundenheit anstelle von Gebundenheit}

Um negative Effekte zu vermeiden und die Kundenbindung zu stärken, plädieren Henderson et al. (2011) dafür, Anreize darauf auszurichten, die Kundenbeziehung von einer reinen Austauschbeziehung auf eine ,höhere Ebene“ zu bringen und die Kundenbindung so zu stärken. Hierbei ist es entscheidend, dem Kunden zu signalisieren, dass es dem Anbieter nicht nur um eine rein ökonomische Geschäftsbeziehung geht. Das Eigeninteresse des Händlers sollte daher nicht offensichtlich sein (Henderson et al. 2011). Insbesondere durch die in Abschn. 5.1 erwähnten Anreizarten bieten sich vielfältige Möglichkeiten, die das Interesse des Händlers am Wohl des Kunden signalisieren, ohne die werbliche Absicht in den Vordergrund zu stellen (Gàzquez-Abad et al. 2011). Derartige Anreize sollten die ,Verbundenheit“ der Konsumenten zum jeweiligen Unternehmen fördern. Sind Anreize auf ein rein ökonomisches Kosten-Nutzen Verhältnis ausgerichtet (z.B. monetäre Anreize), so entsteht zwar „Gebundenheit“, die Einstellung der Konsumenten verändert sich jedoch nicht (Bliemel und Eggert 1998, S. 41-43; Hansen 2011, S. 301). Zwar gelingt es Unternehmen durch solche Massnahmen kurzfristig Wechselbarrieren aufzubauen, das Ziel einer langfristigen Kundenbeziehung verfehlen diese Instrumente jedoch. Besonders problematisch ist hierbei, dass solche Wechselbarrieren schnell wegfallen können und die ,unverbundenen“ Kunden in diesem Fall zur Konkurrenz abwandern (Bendapudi und Berry 1997, S. 28f, S. 32; Fornell 1992, S. 10). Daher sollten Kundenbindungsinstrumente stets darauf abzielen, die Beziehung auf eine höhere Ebene zu bringen und Verbundenheit zu schaffen (Shugan 2005, S. 188).

\subsection{Abstimmung der Anreize auf Situationen und Zielgruppen}

Darüber hinaus verdeutlichen wissenschaftliche Untersuchungen auf diesem Gebiet, dass Kundenbindungsinstrumente je nach Situation, spezifischen Bedingungen und Zielgruppe unterschiedliche Auswirkungen haben (Meyer-Waarden 2007, S. 234). Diese Bedingungen (Moderatoren) gilt es bei der Ausgestaltung der Anreize zu berücksichtigen (vgl. Abschn. 3.4). Bestehende Studien zeigen in diesem Zusammenhang, dass bspw. die Branche, die Markenbekanntheit, das Involvement, die Loyali- 
tät, die Selbstbestimmung, die Zufriedenheit, das Produktinteresse, die Beziehungsstärke und die Kanalpräferenz der Konsumenten die Wirkung der Anreize beeinflussen können. Um den Einsatz wirkungsloser oder sogar schädlicher Instrumente zu vermeiden, gilt es die Wirkung unter den jeweiligen Bedingungen im Vorfeld zu prüfen. Gleichzeitig sollten Kundenbindungsinstrumente nicht für alle Konsumenten gleichermassen eingesetzt werden. Daher gilt es, zunächst die Kundenbasis zu segmentieren und dann entsprechende Zielgruppen auszuwählen (Meyer-Waarden 2007, S. 234). Durch Warenkorbanalysen und Kundenbefragungen sind die zur Segmentierung der Kundenbasis notwendigen Informationen in den meisten Handelsunternehmen heute einfach zugänglich. Zielgruppengerichtete Aktionen sind für den Händler somit günstiger als willkürlich eingesetzte Anreize und erzielen gleichzeitig positivere Wirkungen in Hinblick auf die Einstellungen und das Kaufverhalten (Melancon et al. 2011, S. 357f).

Hinzu kommt, dass die Kombination unterschiedlicher Merkmale (z.B. sofortige monetäre Anreize, verzögerte nicht-monetäre Anreize) Wechselwirkungen hervorrufen kann. Während einige Kombinationen besonders vorteilhaft sind (z.B. anbieterbezogene sofortige Anreize), sind andere Kombinationen weniger vorteilhaft (z.B. anbieterbezogene verzögerte Anreize, Tietje 2002). Auch diese Interaktionen gilt es bei der Ausgestaltung der Anreize zu beachten, um eine grösstmögliche Effektivität sicherzustellen und negative Auswirkungen zu vermeiden.

\subsection{Effizienter Einsatz von Kundenbindungsinstrumenten}

Grundsätzlich ist eine positive Beeinflussung des Unternehmenserfolgs durch eine verbesserte Kundenbindung anzunehmen (Bruhn 2009, S. 67; Krafft und Götz 2011, S. 215f; Leusser et al. 2011, S. 22; Müller 2006, S. 195-215; Reinartz et al. 2004, S. 299, S. 301). Dieser positive Effekt wird bspw. mit einer höheren Kundenpenetration, einer grösseren Preistoleranz der Kunden, einer stärkeren Weiterempfehlungsbereitschaft und einer damit einhergehenden besseren Ausschöpfung des Absatzund Umsatzpotenzials begründet (Diller 1997, S. 40; Diller 2011, S. 251; Goerdt 1999, S. 284; Uncles et al. 2003, S. 303). Da Unternehmensaktivitäten zur Erhöhung der Kundenbindung jedoch stets auch mit Kosten verbunden sind, gilt es, den Einsatz von Kundenbindungsinstrumenten kritisch aus Kosten-Nutzen-Gesichtspunkten zu beleuchten (Diller 2011, S. 250; Krüger-Strohmayer 2000, S. 233-241; Rust et al. 2004, S. 112; Uncles et al. 2003, S. 303). Spezifische Kundenbindungskosten und potenzielle negative Effekte der Kundenbindung wie Reaktanzeffekte und Flexibilitätseinbussen des Unternehmens spielen hierbei eine Rolle (Diller 2011, S. 262; Shugan 2005, S. 186; Uncles et al. 2003, S. 307). Untersuchungen bestätigen, dass sich eine höhere Kundenbindung nicht zwangsläufig in verbesserten ökonomischen Kennzahlen niederschlägt (z.B. Reinartz und Krafft 2001; Reinartz und Kumar 2000). So kommen bspw. Reinartz und Krafft (2001) zu dem Ergebnis, dass aus Anbietersicht sehr profitable Kundensegmente existieren, die sich durch eine geringe Kundenbindung, d.h. ein eher transaktionales Beziehungsverhalten, auszeichnen (Reinartz und Krafft 2001, S. 1275). Auch Leenheer et al. (2007) haben gezeigt, dass die Mitgliedschaft in einem Loyalitätsprogramm nur einen geringen Einfluss auf das Kaufverhalten (Share of Wallet) von Kunden unterschiedlicher Anbieter hat. Dieser Effekt wird häufig überschätzt, da die Teilnehmer an 
Loyalitätsprogrammen oftmals ohnehin treue Kunden sind. Ein weiterer Grund hierfür ist, dass Loyalitätsprogramme die Kunden zwar häufig an das Programm, jedoch nicht unbedingt an das Unternehmen binden (z.B. Dowling und Uncles 1997; Evanschitzky et al. 2012; Meyer-Waarden 2007; Yi und Jeon 2003). Sind Kunden einem Anbieter nur so lange treu, wie sie auch einen Anreiz für ihre Treue erhalten, so entsteht keine nachhaltig effiziente Kundenbindung (Evanschitzky et al. 2012; Meyer-Waarden 2007, S. 234).

Generell finden sich in der wissenschaftlichen Literatur bisher vergleichsweise wenige Studien, die sich mit der Effizienz konkreter Kundenbindungsinstrumente beschäftigen (Krafft und Götz 2011, S. 228). Eine dieser Untersuchungen von Rust und Verhoef (2005) zeigt empirisch, dass sich der Unternehmensgewinn durch einen zielgruppenspezifischen Einsatz monetärer und nicht-monetärer Anreize positiv beeinflussen lässt. Reutterer et al. (2006) bestätigen dieses Ergebnis und zeigen gleichzeitig, dass negative Effekte auf den Unternehmensgewinn entstehen, wenn Anreize willkürlich, d.h. nicht zielgruppengerichtet, zum Einsatz kommen.

Für die Praxis gilt, dass der Einsatz von Kundenbindungsinstrumenten stets einem kritischen Erfolgscontrolling zu unterziehen ist (Bruhn 2009, S. 275; Goerdt 1999, S. 280f). Im Idealfall sollte ein solches Erfolgscontrolling sowohl einstellungsbezogene Erfolgskennzahlen (z.B. Steigerung der Kundenzufriedenheit) als auch tatsächliche Umsatz- und Kostenkennzahlen berücksichtigen. Entscheidend ist zudem, die gegebenenfalls erzielten Umsatzeffekte auf Neu- und Bestandskunden aufzuteilen (z.B. Rust et al. 2004, S. 112). Während bestimmte Instrumente evtl. sporadisch neue Kunden anlocken und somit eine kurzfristige Umsatzsteigerung bewirken, leisten sie nicht zwangsläufig auch einen Beitrag zur Bindung bestehender Kunden. Für die Umsetzung eines solchen Erfolgscontrollings sind insbesondere die Marktforschungsabteilungen in den Unternehmen gefragt. Entsprechend der Ergebnisse derartiger Analysen sind der Einsatz und die Ausgestaltung der Anreize gegebenenfalls anzupassen. Auch in der wissenschaftlichen Forschung wäre eine stärkere Berücksichtigung echter Erfolgskennzahlen in Zukunft wünschenswert (vgl. Abschn. 6.1.1).

\section{Ausblick und Fazit}

\subsection{Zukünftige Forschungsarbeiten zum Einsatz von Kundenbindungsinstrumenten}

Aus den Ergebnissen der aufgezeigten Studien lassen sich relevante Hinweise für künftige Forschungsarbeiten ableiten. Wir wollen künftige Forscher motivieren, insbesondere die folgenden Aspekte in Zukunft verstärkt zu bearbeiteten:

1. Einbezug echter Verkaufszahlen im Gegensatz zur bisherigen überwiegenden Fokussierung auf Einstellungen und Intentionen

2. Berücksichtigung zusätzlicher Moderatoren der Wirkung von Kundenbindungsinstrumenten auf Einstellungen und Kaufverhalten

3. Gezielte Analyse von Konsumentengruppen, die keinen Anreiz erhalten

4. Weitere Differenzierung der Ausprägungen unterschiedlicher Anreize

5. Kreuzvalidierung der empirischen Ergebnisse in 
- unterschiedlichen Branchen

- unterschiedlichen Ländern

6. Vertiefende Untersuchung firmenspezifischer und firmenübergreifender Anreize

7. Empirische Untersuchungen zur Effizienz von

- Loyalitätsprogrammen im Allgemeinen

- spezifischen Kundenbindungsinstrumenten

Auf jede dieser Forderungen an künftige Forschungsarbeiten gehen wir im Folgenden ein und beschreiben mögliche Vorgehensweisen derartiger Forschungsprojekte.

\subsubsection{Einbezug echter Verkaufszahlen}

Der Grossteil der aufgezeigten Studien analysierte die Kundenbindungsinstrumente im Rahmen von Laborexperimenten oder Befragungen. Das Kaufverhalten wird dabei mit Hilfe von Indikatoren zur Messung von Verhaltensintentionen erfasst.

Indirekt unterstellen diese Studien, dass sich eine positive Beeinflussung der Kundenwahrnehmung im tatsächlichen Kaufverhalten der Kunden niederschlägt. Diese Annahme treffen auch unterschiedliche konzeptionelle Modelle, welche sich generell mit der Effizienz von Marketinginvestitionen befassen. Sie unterstellen meist einen direkten, positiven Zusammenhang zwischen Einstellungen und Verhalten (z.B. Bolton et al. 2004, S. 4; Lemon et al. 2001, S. 22f; Rust et al. 2004, S. 112). Allerdings haben empirische Untersuchungen gezeigt, dass sich positive Wahrnehmungen, Einstellungen und Intentionen nicht immer in tatsächlichem Kaufverhalten und folglich im Umsatz niederschlagen (z.B. Mittal und Kamakura 2001; Seiders et al. 2005; Voss et al. 2010). Seiders et al. (2005) fanden bspw. heraus, dass Wahrnehmungen und Einstellungen der Konsumenten Variationen im tatsächlichen Kaufverhalten nur zu $10 \%$ erklären. Evanschitzky et al. (2012) zeigen, dass weniger als $8 \%$ des tatsächlichen Kaufverhaltens durch die einstellungsbezogene Loyalität zum Anbieter erklärt werden. ${ }^{1}$ Auch Untersuchungen von Vogel et al. (2008) und Voss et al. (2010) bestätigen, dass der Effekt von Einstellungen und Intentionen auf das tatsächliche Kaufverhalten häufig gering ausfällt. Die positive Beeinflussung des Kaufverhaltens ist, neben der Beeinflussung der Einstellungen, jedoch ein wesentliches Ziel, das Anbieter mit dem Einsatz von Kundenbindungsinstrumenten verfolgen. Das bedeutet, dass die Kundenbindung sowohl eine intentionale Einstellungskomponente als auch eine faktische Verhaltenskomponente enthält (Bruhn 2009, S. 263f; Homburg und Bruhn 2010, S. 6-10). Obwohl die Unterscheidung zwischen intentionaler und faktischer Kundenbindung in der Wissenschaft nicht neu ist, wurde das tatsächliche Kaufverhalten in der Forschung bisher vernachlässigt. Ein Grossteil der aufgezeigten Studien untersucht den Effekt von Kundenbindungsinstrumenten auf Kaufintentionen oder Einstellungen und betrachtet somit nur die intentionale Kundenbindung. Insbesondere die Effizienz der Instrumente lässt sich so nicht bewerten, da - gemäss der genannten Studien - mehr als $90 \%$ der Variation im tatsächlichen Kaufverhalten

\footnotetext{
${ }^{1}$ Ca. $8 \%$ der Varianz im Kaufverhalten werden durch die einstellungsbezogene Loyalität zum Anbieter und die Loyalität zum Kundenbindungsprogramm erklärt (Evanschitzky et al. 2012).
} 
durch andere Grössen beeinflusst werden. Daher sollten künftige Forschungsarbeiten verstärkt echte Anreize sowie Verhaltensdaten in die Analyse einbeziehen. Hier$\mathrm{zu}$ bieten sich Feldexperimente an, welche unterschiedliche Anreize an bestimmte Konsumenten verschicken. Durch die Kombination von Einstellungen (z.B. durch Befragungen) und tatsächlichem Kaufverhalten (z.B. über Kundenkartendaten) sind validere Aussagen über die tatsächliche Wirkung der Anreize möglich. Hierdurch werden Verzerrungen durch die isolierte Messung von Intentionen (z.B. Common Method Bias) vermieden und die externe Validität erhöht (Malhotra und Birks 2007; Mittal und Kamakura 2001; Podsakoff et al. 2003).

\subsubsection{Berücksichtigung zusätzlicher Moderatoren}

Künftige Forschungsarbeiten sollten ausserdem weitere Moderatoren der Wirkung von Kundenbindungsinstrumenten auf die Einstellungen und das Kaufverhalten einbeziehen. Dies könnten bspw. persönliche Charakteristika der Konsumenten sowie situative Faktoren sein. Zudem sollten auch Umsatzkennzahlen als Moderatoren dienen. Denkbar wäre ein experimentelles Design, welches unterschiedliche Kundensegmente (z.B. A-, B- und C-Kunden) mit unterschiedlichen Anreizen bearbeitet und Einstellungs- sowie Verhaltensreaktionen dieser Segmente erfasst (Rust und Verhoef 2005). Darüber hinaus sollten künftige Forschungsprojekte die Wirkung von Kundenbindungsanreizen für Anbieter mit unterschiedlichen Geschäftsmodellen analysieren. Möglicherweise wirken monetäre Anreize für einen preisorientierten Anbieter anders auf die Einstellungen und das Kaufverhalten als für einen qualitäts- oder serviceorientierten Anbieter.

\subsubsection{Analyse von Konsumenten, die keinen Anreiz erhalten}

Bisherige Studien fokussieren sich ausschliesslich auf die Wirkung von Kundenbindungsanreizen auf die jeweils ausgewählten Zielgruppen. Eine weitere interessante Thematik für künftige Forschungsprojekte wäre jedoch auch die Wirkung unterschiedlicher Anreize auf Konsumenten, die keinen Anreiz erhalten (Henderson et al. 2011). Studien zu Preis-Promotions im Allgemeinen zeigen bspw., dass zielgruppenspezifische Preisnachlässe negative Effekte auf die Emotionen und Einstellungen derjenigen Konsumenten ausüben können, die keinen Nachlass erhalten (z.B. Darke und Dahl 2003; Feinberg et al. 2002; Gelbrich 2011): „Few things stir up a consumer revolt quicker than the notion that someone else is getting a better deal" (Feinberg et al. 2002, S. 277). Da sich Konsumenten (z.B. über das Internet) immer stärker untereinander austauschen, nimmt die Gefahr, dass sich diejenigen ohne Anreiz diskriminiert fühlen, zu (Hansen 2011, S. 308). Diese Konsumenten sollten im Rahmen eines Experiments nicht nur als Kontrollgruppe dienen, sondern eine konkrete Experimentalgruppe darstellen. Hierfür könnten Konsumenten, die keinen Anreiz erhalten haben, explizit danach befragt werden, ob Nachbarn, Freunde oder Bekannte aktuell einen Anreiz von einem Anbieter erhalten haben und um welchen Anreiz es sich hierbei handelt. Anschliessend gilt es, die Emotionen, Einstellungen und das Verhalten dieser Kunden messen. 


\subsubsection{Weitere Differenzierung der Anreize}

Darüber hinaus sollten künftige Arbeiten eine weitere Differenzierung der Kundenbindungsanreize vornehmen. Beispielsweise könnte die Wirkung unterschiedlicher monetärer Anreize für bestimmte Zielgruppen oder in verschiedenen Branchen analysiert werden. Die unterschiedlichen monetären Anreize sollten bspw. in ihrer Höhe und in ihrer Kommunikation variieren. Möglicherweise lässt sich eine kritische Schwelle feststellen, an der sich die betrachteten Effekte (z.B. positiver Effekt auf die Einstellungen) ins Negative umkehren. Ebenso könnte sich eine bestimmte Kommunikationsart als überlegen herausstellen. Auch könnten künftige Studien eher sozial ausgerichtete nicht-monetäre Anreize (z.B. Einladungen zu sozialen Events) mit weniger sozialen nicht-monetären Anreizen vergleichen.

\subsubsection{Kreuzvalidierung von Ergebnissen}

Ein weiteres wichtiges Feld für künftige Forschungsarbeiten sind länder- und branchenübergreifende Studien. Wie de Wulf et al. (2001) gezeigt haben, rufen die von ihnen untersuchten Instrumente in drei unterschiedlichen Ländern und zwei unterschiedlichen Branchen teilweise unterschiedliche Wirkungen hervor. Das bedeutet, dass die Ergebnisse bestehender Studien nicht immer uneingeschränkt auf andere Länder und Branchen übertragbar sind. Sie sind daher jeweils im spezifischen Kontext zu werten bzw. nur auf Branchen und Länder mit ähnlichen Eigenschaften zu übertragen. Dies betrifft bei den Branchen bspw. die Art der Produkte (z.B. Komplexität, utilitarisitsche vs. hedonistische Güter, Schnelllebigkeit), die Wettbewerbssituation uns die Marktsättigung. In Hinblick auf die Länder sind zum einen generelle kulturelle Aspekte, welche die Reaktion der Konsumenten auf Kundenbindungsinstrumente beeinflussen, von Bedeutung. Zum anderen spielt es eine Rolle, wie weit der Marketingfortschritt in dem jeweiligen Land ist und inwiefern die Konsumenten bereits mit bestimmten Instrumenten vertraut sind. Künftige Forschungsarbeiten sollten sich gezielt mit dieser Thematik befassen. Um die Generalisierbarkeit der Ergebnisse zu erhöhen, sollten künftige Arbeiten Kreuzvalidierungen der Ergebnisse in unterschiedlichen Ländern und Branchen vornehmen. Hierzu gibt es bisher noch kaum empirische Ergebnisse.

\subsubsection{Vertiefende Untersuchung von firmenspezifischen und firmenübergreifenden Anreizen}

Ein relativ neues Forschungsfeld stellt die Analyse der Wirkung von firmenspezifischen und firmenübergreifenden Anreizen dar (Evanschitzky et al. 2012; Hartmann 2001; Müller 2006). Lemon und von Wangenheim (2009) haben gezeigt, dass firmenübergreifende Anreize Konsumenten zum Cross-Buying motivieren. Allerdings gibt es in diesem Kontext zahlreiche unbeantwortete Fragen. So sind bspw. Kosteneinsparungsmöglichkeiten durch kooperative Loyalitätsprogramme in künftige Analysen einzubeziehen. Zudem könnten künftige Untersuchungen Imageeffekte auf Unternehmen überprüfen, die durch die Kooperation in firmenübergreifenden Loyalitätsprogrammen entstehen. Labor- und insbesondere Feldexperimente sollten die Effektivität von firmenspezifischen im Vergleich zu firmenübergreifenden Anreizen im 
Hinblick auf die Beeinflussung der Einstellungen und des Kaufverhaltens von Konsumenten überprüfen.

\subsubsection{Empirische Untersuchungen zur Effizienz von Kundenbindungsinstrumenten}

Wie Abschn. 5.5 aufgezeigt hat, existieren bisher kaum empirische Untersuchungen zur Effizienz von Loyalitätsprogrammen und konkreten Kundenbindungsanreizen. Ein Grund hierfür ist die mangelnde Verfügbarkeit von Umsatz- und Kostendaten, die sich den jeweiligen Kundenbindungsmassnahmen zuordnen lassen. Gerade vor dem Hintergrund, dass Unternehmen in enormem Masse in Loyalitätsprogramme investieren, drängen sich vertiefende Analysen zu diesem Thema auf. Wie der Literaturüberblick gezeigt hat, zeigen sich häufig keine Effekte der eingesetzten Anreize auf die abhängigen Erfolgsgrössen und die Effizienz ist somit fraglich. Dies mag u.a. an der in Abschn. 6.1.1 angesprochenen fehlenden Verhaltenswirksamkeit der eingesetzten Instrumente liegen. Gleichzeitig können jedoch auch wirkungsvolle Instrumente ein unverhältnismässiges Kosten-Nutzen-Verhältnis aufweisen. Hierzu existieren kaum empirische Untersuchungen. Daher bieten sich bspw. firmenübergreifende Untersuchungen an, welche den Einsatz konkreter Kundenbindungsmassnahmen, den Grad der Kundenbindung und den Unternehmenserfolg in einen Zusammenhang bringen.

\subsection{Zusammenfassung, Limitationen und Fazit}

Wie alle wissenschaftlichen Beiträge hat auch dieser Artikel gewisse Limitationen. Zum einen ist einschränkend anzumerken, dass insbesondere die Ergebnisse älterer Studien nicht eins zu eins auf die heutige Marketingwelt übertragbar sind. Da sowohl Konsumenten als auch Wettbewerber in gewisser Weise auf bestimmte Kundenbindungsinstrumente reagieren, kann sich deren Wirkung im Zeitverlauf verändern (Pauwels 2004). Während bestimmte Instrumente bspw. vor zehn Jahren noch effektiv waren, sind sie heute vielleicht weniger oder gar nicht mehr wirksam. Daher sind die Ergebnisse älterer Studien mit etwas mehr Vorsicht zu bewerten als die Ergebnisse aktueller Untersuchungen. Hinzu kommt, dass die Ergebnisse bestimmter Untersuchungen nicht immer uneingeschränkt auf andere Länder und Branchen übertragbar sind. Sie sind daher im jeweiligen Kontext zu werten.

Trotz dieser Einschränkungen hat der vorliegende Beitrag auf Basis bestehender Forschungsergebnisse gezeigt, warum die immensen Investitionen in Loyalitätsprogramme und Kundenbindungsinstrumente nicht immer wirksam sind. Ein wesentlicher Grund besteht darin, dass Marketingmanager bei der Umsetzung die potenziellen Wirkungsunterschiede von Kundenbindungsinstrumenten unter den jeweiligen Rahmenbedingungen vernachlässigen. Häufig sind die Anreize zu wenig auf die jeweiligen Branchenmerkmale oder auf bestimmte Zielgruppen abgestimmt. Dies führt nicht nur dazu, dass die Instrumente wirkungslos sind; es können sich sogar negative Effekte auf die jeweiligen Zielgrössen ergeben. Ebenso erfolgt der Einsatz von Kundenbindungsinstrumenten in der Praxis häufig zu unsystematisch. Insbesondere Wechselwirkungen zwischen den Merkmalen der Anreize werden zu wenig beachtet. Bestehende Studien zeigen jedoch, dass bestimmte Merkmalskombinationen effizienter sind als andere. In der Praxis könnten Methoden der Marktforschung und des 
Data-Minings helfen, Anreize zielgerichteter anzuwenden und damit die Effizienz zu erhöhen. Ebenso wichtig ist ein kontinuierliches Erfolgscontrolling der eingesetzten Instrumente. Da die meisten Unternehmen heutzutage aufgrund ihrer Loyalitätsprogramme und der vorhandenen Scannerdaten über die entsprechende Datenbasis verfügen, gestaltet sich eine solche Marktforschung sowie das Erfolgscontrolling einfacher als noch vor wenigen Jahren.

\section{Literatur}

Ailawadi KL, Keller KL (2004) Understanding retail branding: conceptual insights and research priorities. J Retail 80(4):331-342

BBDO (2009) Kundenpflege erlebt 2010 eine Renaissance, http://www.bbdo.de/_download/pdf/bbdo_ consulting-studie1.download.Par.0001.Link1Download.File1Title.pdf. Gesehen 23. Februar 2012

Bendapudi N, Berry LL (1997) Customers' motivations for maintaining relationships with service providers. J Retail 73(1):15-37

Berry LL (1995) Relationship marketing of services—growing interest, emerging perspectives. J Acad Mark Sci 23(4):236-245

Bhattacharya CB, Bolton RN (2000) Relationship marketing in mass markets. In: Sheth JN, Parvatiyar A (Hrsg) Handbook of relationship marketing. Sage, Thousand Oaks, S 327-354

Bliemel FW, Eggert A (1998) Kundenbindung - Die neue Sollstrategie? Marketing ZFP 1(1):37-46

Bolton RN (1998) A dynamic model of the duration of the customer's relationship with a continuous service provider: the role of satisfaction. Mark Sci 17(1):45-65

Bolton RN, Lemon KN, Verheof PC (2004) The theoretical underpinnigs of customer asset management: a framework and propositions for future research. J Acad Mark Sci 32(3):271-292

Bowman D, Narayandas D (2001) Managing customer-initiated contacts with manufacturers: the impact on share of category requirements and word-of-mouth behavior. J Mark Res 38(3):281-297

Brodie RJ, Coviello NE, Brookes RW, Little V (1997) Towards a paradigm shift in marketing? An examination of current marketing practices. J Mark Manag 13(5):383-406

Bruhn M (2009) Relationship marketing. Das management von Kundenbeziehungen, 2. Aufl. Vahlen, München

Bruhn M (2011) Zufriedenheits- und Kundenbindungsmanagement. In: Hippner H, Hubrich B, Wilde KD (Hrsg) Grundlagen des CRM - Strategie, Geschäftsprozesse und IT-Unterstützung, 3. Aufl. Gabler, Wiesbaden, S 404-439

Christy R, Oliver G, Penn J (1996) Relationship marketing in consumer markets. J Mark Manag 12(13):175-187

Dabholkar PA, Thorpe DI, Rentz JO (1996) A measure of service quality for retail stores: scale development and validation. J Acad Mark Sci 24(1):3-16

Darke PR, Dahl DW (2003) Fairness and discounts: the subjective value of a bargain. J Consum Psychol 13(3):328-338

Deci EL (1971) Effects of externally mediated rewards on intrinsic motivation. J Pers Soc Psychol 18(1):105-115

Deci EL (1975) Intrinsic motivation. Plenum, New York

Deci EL, Ryan RM (1985) Intrinsic motivation and self-determination in human behavior. Plenum, New York

Deci EL, Ryan RM, Koestner R (1999) A meta-analytic of experiments examining the effects of extrinsic rewards on intrinsic motivation. Psychol Bull 125(6):627-668

Deutscher Dialogmarketingverband (2011) Erfolg durch Dialog, http://www.ddv.de/index.php?id=75. Gesehen 23. Februar 2012

de Wulf K, Odekerken-Schröder G, Iacobucci D (2001) Investments in consumer relationships: a crosscountry and cross-industry exploration. J Mark 54(4):33-50

Dholakia UM (2006) How customer self-determination influences relational marketing outcomes: evidence from longitudinal field studies. J Mark Res 43(1):109-120

Dick A, Basu K (1994) Customer loyalty: toward an integrated conceptual framework. J Acad Mark Sci 22(2):99-113

Diller H (1995) Beziehungs-Marketing. WiSt Wirtschaftswiss Stud 24(9):442-447 
Diller H (1996) Kundenbindung als Marketingziel. Marketing ZFP 18(2):81-94

Diller H (1997) Was leisten Kundenclubs? Marketing ZFP 19(1):33-41

Diller H (2011) Die Bedeutung des Beziehungsmarketing für den Unternehmenserfolg. In: Hippner H, Hubrich B, Wilde KD (Hrsg) Grundlagen des CRM - Strategie, Geschäftsprozesse und ITUnterstützung, 3. Aufl. Gabler, Wiesbaden, S 247-270

Diller H, Haas A, Ivens B (2005) Verkauf und Kundenmanagement - Eine prozessorientierte Konzeption. Kohlhammer, Stuttgart

Dowling GR, Uncles M (1997) Do customer loyalty programs really work? MIT Sloan Manag Rev 38(4):71-82

Evanschitzky H, Ramaseshan B, Woisetschläger DM, Richelsen V, Blut M, Backhaus C (2012) Consequences of customer loyalty to the program and to the company. J Acad Mark Sci 40(5):625-638

Fehr E, Falk A (2002) Psychological foundations of incentives. Eur Econ Rev 46(4-5):687-724

Feinberg FM, Krishna A, Zhang ZJ (2002) Do we care what others get? A behaviorist approach to targeted promotions. J Mark Res 39(3):277-291

Ferguson R, Hlavinka K (2007) The COLLOQUY loyalty marketing census: sizing up the US loyalty marketing industry. J Consum Mark 24(5):313-321

Ferguson R, Hlavinka K (2009) In: The big sort-the 2009 COLLOQUY loyalty marketing census. Colloquy talk, Cincinnati

Fornell C (1992) A national customer satisfaction barometer: the Swedish experience. J Mark 56(1):6-21

Gàzquez-Abad JC, de Canniére MH, Martinez-Lopez FJ (2011) Dynamics of customer response to promotional and relational direct mailings from an apparel retailer: the moderating role of relationship strength. J Retail 87(2):166-181

Gelbrich K (2011) I have paid less than you! The emotional and behavioral consequences of advantaged price inequality. J Retail 87(2):207-224

Godfrey A, Seiders K, Voss GB (2011) Enough is enough! The fine line in executing multichannel relational communication. J Mark 75(4):94-109

Georgi D, Mink M (2011) Konzeption von Kundenbeziehungsstrategien. In: Hippner H, Hubrich B, Wilde KD (Hrsg) Grundlagen des CRM - Strategie, Geschäftsprozesse und IT-Unterstützung, 3. Aufl. Gabler, Wiesbaden, S 57-89

Goerdt T (1999) Die Marken- und Einkaufsstättentreue der konsumenten als Bestimmungsfaktoren des vertikalen Beziehungsmarketing. GIM, Nürnberg

Gomez MI, McLaughlin EW, Wittink DR (2004) Customer satisfaction and retail performance: an empirical investigation. J Retail 80(4):265-278

Graham JF (1994) Increasing repurchase rates: a reappraisal of coupon effects. Psychol Mark 11(6):533548

Grewal D, Levy M, Kumar V (2009) Customer experience management in retailing: an organizing framework. J Retail 85(1):1-14

Grönroos C (1996) Relationship marketing: strategic and tactical implications. Manag Decis 34(3):5-14

Grönroos C (1999) Relationship marketing: challenges for the organization. J Bus Res 46(3):327-335

Hansen U (2011) Beziehungslos im Dschungel des Beziehungsmarketing oder: Grenzen des Beziehungsmarketing aus Verbraucherperspektive. In: Hippner H, Hubrich B, Wilde KD (Hrsg) Grundlagen des CRM - Strategie, Geschäftsprozesse und IT-Unterstützung, 3. Aufl. Gabler, Wiesbaden, S 294-315

Hartmann T (2001) A new paradigm of customer management: why multipartner strategies will create more value for consumers and industry. Eur Retail Dig 9(31):12-14

Heiligenthal J, Skiera B (2007) Optimale Verteilung eines Budgets auf Aktivitäten zur Kundenakquisition, Kundenbindung und Add-on-Selling. ZfB Z Betriebswirtsch (Special issue) 3:117-141

Helm R, Ludl M (2005) Kundenkarten und ihre Wirkung als Kundenbindungsinstrument. Z Betriebswirtsch 75(12):1131-1163

Henderson CM, Beck JT, Palmatier RW (2011) Review of the theoretical underpinnings of loyalty programs. J Consum Psychol. doi:10.1016/j.jcps.2011.02.007

Hennig-Thurau T, Hansen U (2000) Relationship marketing-some reflections on the state-of-the-art of relational concept. In: Hennig-Thurau H, Hansen U (Hrsg) Relationship marketing: gaining competitive advantage through customer satisfaction and retention. Springer, Berlin, S 3-27

Hennig-Thurau T, Paul M (2007) Can economic bonus programs jeopardize service relationships. Serv Bus 1(2):159-175

Hesse J, Krafft M, Peters K (2007) Boarderless direct marketing? In: Krafft M, Hesse J, Höfling J, Peters K, Rinas D (Hrsg) International direct marketing_principles, best practices, marketing facts. Springer, Berlin, S 3-40 
Homburg C, Bruhn M (2010) Kundenbindungsmanagement - Eine Einführung in die theoretischen und praktischen Problemstellungen. In: Bruhn M, Homburg C (Hrsg) Handbuch Kundenbindungsmanagement. Gabler, Wiesbaden, S 3-39

Homburg C, Fassnacht M (2001) Kundennähe, Kundenzufriedenheit und Kundenbindung. In: Bruhn M, Meffert H (Hrsg) Handbuch Dienstleistungsmanagement. Gabler, Wiesbaden, S 441-463

Homburg C, Giering A (2001) Personal characteristics as moderators of the relationship between customer satisfaction and loyalty - an empirical analysis. Psychol Mark 18(1):43-66

Jones MA, Mothersbaugh DL, Beatty SE (2000) Switching barriers and repurchase intentions in services. J Retail 76(2):109-126

Keh HT, Lee YH (2006) Do reward programs build loyalty for services? The moderating effect of satisfaction on type and timing of rewards. J Retail 82(2):127-136

Krafft M, Götz O (2011) Der Zusammenhang zwischen Kundennähe, Kundenzufriedenheit und Kundenbindung sowie deren Erfolgswirkungen. In: Hippner H, Hubrich B, Wilde KD (Hrsg) Grundlagen des CRM - Strategie, Geschäftsprozesse und IT-Unterstützung, 3. Aufl. Gabler, Wiesbaden, S 213-246

Krüger-Strohmayer S (2000) Profitabilitätsorientierte Kundenbindung durch Zufriedenheitsmanagement kundenzufriedenheit und Kundenwert als Steuerungsgrösse für die Kundenbindung in marktorientierten Dienstleistungsunternehmen. FGM-Verlag, München

Kumar V, George M (2007) Measuring and maxamizing customer equity: a critical analysis. J Acad Mark Sci 35(2):157-171

Kumar V, Reinartz WJ (2006) Customer relationship management-a databased approach. Wiley, Hoboken

Kumar V, Shah D (2004) Building and sustaining profitable customer loyalty for the 21st century. J Retail 80(4):317-330

Kunkel JH, Berry LL (1968) A behavioral conception of retail image. J Mark 32(4):21-27

Lal R, Bell DE (2003) The impact of frequent shoper programs in grocery retailing. Quant Mark Econ 1(2):179-202

Leenheer J, van Heerde HJ, Bijmolt THA, Smidts A (2007) Do loyalty programs really enhance behavioral loyalty? An empirical analysis accounting for self-selecting members. Int J Mark Res 24(1):31-47

Lemon KN, von Wangenheim F (2009) The reinforcing effects of loyalty program partnerships and core service usage. J Serv Res 11(4):357-370

Lemon KN, Rust RT, Zeithaml VA (2001) What drives customer equity? Mark Manag 10(1):20-25

Leusser W, Hippner H, Wilde KD (2011) CRM - Grundlagen, Konzepte und Prozesse. In: Hippner H, Hubrich B, Wilde KD (Hrsg) Grundlagen des CRM - Strategie, Geschäftsprozesse und IT-Unterstützung, 3. Aufl. Gabler, Wiesbaden, S 16-55

Lewis M (2004) The influence of loyalty programs and short-term promotions on customer retention. J Mark Res 41(3):281-292

Liu Y (2007) The long-term impact of loyalty programs on consumer purchase behavior and loyalty. J Mark 71(4):19-35

Liu Y, Yang R (2009) Competing loyalty programs: impact of market saturation, market share, and category expandability. J Mark 73(1):93-108

Mägi AW (2003) Share of wallet in retailing: the effects of customer satisfaction, loyalty cards and shopper characteristics. J Retail 79(2):97-106

Mann A (2007) Dialogmarketing-Kompetenz von Unternehmen: konzeptionelle Überlegungen und empirische Befunde. ZfB Z Betriebswirtschaft (Special Issue) 3:1-27

Meyer-Waarden L (2007) The effects of loyalty programs on customer lifetime duration and share of wallet. J Retail 83(2):223-236

Meyer-Waarden L, Benavent C (2009) Grocery retail loyalty program effects: self-selection or purchase behavior change? J Acad Mark Sci 37(3):345-358

McDonald WJ (1998) Direct marketing — an integrated approach. McGraw Hill, Boston

Melancon JP, Noble SM, Noble CH (2011) Managing rewards to enhance relational worth. J Acad Mark Sci 39(3):341-362

Mittal V, Kamakura WA (2001) Satisfaction, repurchase intent, and repurchase behavior: investigating the moderating effect of customer characteristics. J Mark Res 38(1):131-142

Malhotra NK, Birks DF (2007) Marketing research: an applied approach, 3. Aufl. Pearson, Harlow

Morgan RM, Hunt SD (1994) The commitment-trust theory of relationship marketing. J Mark 58(3):20-38

Müller S (2006) Bonusprogramme als Instrumente des Beziehungsmarketing: Eine theoretische und empirische Analyse. GIM, Nürnberg 
NRF, KPMG (2011) Retail horizons: benchmarks for 2010. Forecasts for 2011, Executive summary, http://www.kpmg.com/US/en/IssuesAndInsights/ArticlesPublications/Documents/Retail\%20 Horizons\%20-\%20Executive\%20Summary\%202011[1].pdf. Gesehen 23. Februar 2012

Nunes JC, Dréze X (2006) Your loyalty program is betraying you. Harv Bus Rev 84(4):124-131

O’Malley L (1998) Can loyalty schemes really build loyalty? Mark Intell Plann 16(1):47-57

O’Malley L, Tyann C (2000) Relationship marketing in consumer markets—rhetoric or reality? Eur J Mark 34(7):797-815

Palmatier RW, Dant RP, Grewal D, Evans KR (2006) Factors influencing the effectiveness of relationship marketing: a meta-analysis. J Mark 70(4):136-153

Pan Y, Zinkhan GM (2006) Determinants of retail patronage: a meta-analytical perspective. J Retail 83(3):229-243

Parvatiyar A, Sheth JN (2001) Conceptual framework of customer relationship management: customer relationship management. In: Sheth JN, Parvatiyar A, Shainesh G (Hrsg) Emerging concepts, tools and applications. Tata McGraw Hill, New Delhi, S 3-25

Pauwels K (2004) How dynamic consumer response, competitor response, company support, and company inertia shape long-term marketing effectiveness. Mark Sci 23(4):596-610

Payne A, Frow P (2005) A strategic framework for customer relationship management. J Mark 69(4):167176

Podsakoff PM, MacKenzie SB, Lee J, Podsakoff NP (2003) Common method biases in behavioral research: a critical review of the literature and recommended remedies. J Appl Psychol 88(5):879-903

Puchner G (2011) Kundenbindung durch Relationship Marketing-Instrumente. EUL Verlag, Lohmar

Reinartz WJ (2010) Understanding customer loyalty programs. In: Krafft M, Matrala MK (Hrsg) Retailing in the 21st century: current and future trends. Springer, Heidelberg, S 409-427

Reinartz WJ, Krafft M (2001) Überprüfung des Zusammenhangs von Kundenbindungsdauer und Kundenertragswert. Z Betriebswirtsch 71(11):1263-1281

Reinartz WJ, Kumar V (2000) On the profitability of long-life customers in a noncontractual setting: an empirical investigation and implications for marketing. J Mark 64(4):17-35

Reinartz WJ, Krafft M, Hoyer WD (2004) The customer relationship management process: its measurement and impact on performance. J Mark Res 41(3):293-305

Reutterer T, Mild A, Natter M, Taudes A (2006) A dynamic segmentation approach for targeting and customizing direct marketing campaigns. J Interact Mark 20(3-4):43-57

Rigby DK, Reichheld FF, Schefter P (2002) Avoid the four perils of CRM. Harv Bus Rev 80(2):101-109

Roehm ML, Pullins EB, Roehm HA (2002) Designing loyalty-building programs for packaged goods brands. J Mark Res 39(2):202-213

Rothschild ML, Gaidis WC (1981) Behavioral learning theory: its relevance to marketing and promotions. J Mark 45(2):70-78

Rust RT, Verhoef PC (2005) Optimizing the marketing interventions mix in intermediate-term CRM. Mark Sci 24(3):477-489

Rust RT, Zeithaml VA, Lemon KN (2000) Driving customer equity-how customer lifetime value is reshaping corporate strategy. The Free Press, New York

Rust RT, Lemon KN, Zeithaml VA (2004) Using customer equity to focus marketing strategy. J Mark 68(1):109-127

Ryan RM, Deci EL (2002) Overview of self-determination theory: an organismic dialectical perspective. In: Deci EL, Ryan RM (Hrsg) Handbook of self-determination research. University Rochester Press, Rochester, S 3-33

Ryals L, Payne A (2001) Customer relationship management in financial services: towards informationenabled relationship marketing. J Strat Mark 9(1):1-25

Ryan RM, Mims V, Koestner R (1983) Relation of reward contingency and interpersonal context to intrinsic motivation: a review and test using cognitive evaluation theory. J Pers Soc Psychol 45(4):736-750

Schweizer Direktmarketing Verband (2011). http://www.sdv-asmd.ch/index.php?TPL=113\&x270_id=284 Gesehen 23. Februar 2012

Seiders K, Voss GB, Grewal D, Godfrey AL (2005) Do satisfied customers buy more? Examining moderating influences in a retailing context. J Mark 69(4):26-43

Sharp B, Sharp A (1997) Loyalty programs and their impact on repeat-purchase loyalty patterns. Int J Mark Res 14(5):473-486

Shugan S (2005) Brand loyalty programs: are they shams? Mark Sci 24(2):185-193

Taylor GA, Neslin SA (2005) The current and future sales impact of a retail frequency reward program. J Retail 81(4):293-305 
Tietje BC (2002) When do rewards have enhancement effects? An availability valence approach. J Consum Psychol 12(4):363-373

Tomczak T, Reinecke S, Dittrich S (2005) Kundenbindung durch Kundenkarten und -clubs. In: Bruhn M (Hrsg) Handbuch Kundenbindung. Gabler, Wiesbaden, S 275-296

Uncles MD, Dowling GR, Hammond K (2003) Customer loyalty and customer loyalty programs. J Consum Mark 20(4):294-316

Vallerand RJ, Ratelle CF (2002) Intrinsic and extrinsic motivation: a hierarchical model. In: Deci EL, Ryan RM (Hrsg) Handbook of self-determination research. University of Rochester Press, Rochester, S 3764

Verhoef PC (2003) Understanding the effect of customer relationship management efforts on customer retention and customer share development. J Mark 67(4):30-45

Vogel V, Evanschitzky H, Ramaseshan B (2008) Customer equity drivers and future sales. J Mark 72(6):98-108

Voss GB, Godfrey A, Seiders K (2010) How complementarity and substitution alter the customersatisfaction-repurchase link. J Mark 74(6):111-127

White RW (1959) Motivation reconsidered: the concept of competence. Psychol Rev 66(5):297-333

Yi Y, Jeon H (2003) Effects of loyalty programs on value perception, program loyalty and brand loyalty. J Acad Mark Sci 31(3):229-240

Zhang ZJ, Krishna A, Dhar SK (2000) The optimal choice of promotional vehicles: front loaded or rearloaded incentives? Manag Sci 64(3):348-362 